\title{
SHARP HARDY-LERAY INEQUALITY FOR CURL-FREE FIELDS WITH A REMAINDER TERM
}

\section{NAOKI HAMAMOTO AND FUTOSHI TAKAHASHI}

\begin{tabular}{|c|l|}
\hline Citation & OCAMI Preprint Series \\
\hline Issue Date & 2020 \\
\hline Type & Preprint \\
\hline Textversion & Author \\
\hline Rights & For personal use only. No other uses without permission. \\
\hline Relation & $\begin{array}{l}\text { The following article has been submitted to Journal of Functional Analysis. After } \\
\text { it is published, it will be found at https://doi.org/10.1016/j.jfa.2020.108790 . }\end{array}$ \\
\hline
\end{tabular}

From: Osaka City University Advanced Mathematical Institute http://www.sci.osaka-cu.ac.jp/OCAMI/publication/preprint/preprint.html 


\title{
SHARP HARDY-LERAY INEQUALITY FOR CURL-FREE FIELDS WITH A REMAINDER TERM
}

\author{
NAOKI HAMAMOTO AND FUTOSHI TAKAHASHI
}

\begin{abstract}
In this paper, we give a new and a simpler approach to the result in []] concerning the best constant of Hardy-Leray inequality for curl-free fields. As a by-product, we obtain an improved inequality with a remainder term. The non-attainability of the best constant is an easy consequence of the new inequality. The proof is based on a decomposition of curl-free fields into radial and spherical parts.
\end{abstract}

\section{INTRODUCTION}

In this paper, we concern the classical functional inequality called the HardyLeray inequality for smooth vector fields and its improvement.

Let $N \in \mathbb{N}$ be an integer with $N \geq 2$ and put $\boldsymbol{x}=\left(x_{1}, \cdots, x_{N}\right) \in \mathbb{R}^{N}$. In the following, $C_{c}^{\infty}(\Omega)^{N}$ denotes the set of smooth vector fields

$$
\boldsymbol{u}=\left(u_{1}, u_{2}, \cdots, u_{N}\right): \Omega \ni \boldsymbol{x} \mapsto \boldsymbol{u}(\boldsymbol{x}) \in \mathbb{R}^{N}
$$

having compact supports on an open subset $\Omega$ of $\mathbb{R}^{N}$.

Let $\gamma$ be a real number. Then it is well known that

$$
\left(\gamma+\frac{N}{2}-1\right)^{2} \int_{\mathbb{R}^{N}} \frac{|\boldsymbol{u}|^{2}}{|\boldsymbol{x}|^{2}}|\boldsymbol{x}|^{2 \gamma} d x \leq \int_{\mathbb{R}^{N}}|\nabla \boldsymbol{u}|^{2}|\boldsymbol{x}|^{2 \gamma} d x
$$

holds for any vector field $\boldsymbol{u} \in C_{c}^{\infty}\left(\mathbb{R}^{N}\right)^{N}$, as far as the integral on the left-hand side is finite (or equivalently $\boldsymbol{u}(\mathbf{0})=\mathbf{0}$ for $\gamma \leq 1-N / 2$ ). This was first proved by J. Leray [II)] when the weight $\gamma=0$, see also the book by Ladyzhenskaya [9]. It is also known that the constant $\left(\gamma+\frac{N}{2}-1\right)^{2}$ is sharp and never attained by any non-zero vector field.

In [Z] , Costin and Maz'ya proved that if the smooth vector fields $\boldsymbol{u}$ are axisymmetric and subject to the divergence-free constraint $\operatorname{div} \boldsymbol{u} \equiv 0$, then the constant $\left(\gamma+\frac{N}{2}-1\right)^{2}$ can be improved and replaced by a larger one. More precisely, they proved the following:

Theorem A. (Costin-Maz'ya [8]) Let $N \geq 2$. Let $\gamma \neq 1-N / 2$ be a real number and $\boldsymbol{u} \in C_{c}^{\infty}\left(\mathbb{R}^{N}\right)^{N}$ be an axisymmetric divergence-free vector field. (If $N=2$, the axisymmetric assumption is not needed). Assume that $\boldsymbol{u}(\mathbf{0})=\mathbf{0}$ if $\gamma<1-N / 2$. Then

$$
C_{N, \gamma} \int_{\mathbb{R}^{N}} \frac{|\boldsymbol{u}|^{2}}{|\boldsymbol{x}|^{2}}|\boldsymbol{x}|^{2 \gamma} d x \leq \int_{\mathbb{R}^{N}}|\nabla \boldsymbol{u}|^{2}|\boldsymbol{x}|^{2 \gamma} d x
$$

Date: June 17, 2020.

1991 Mathematics Subject Classification. Primary 26D10; Secondary 35A23.

Key words and phrases. Hardy-Leray inequality, curl-free vector fields, remainder term, the best constant. 
holds with the optimal constant $C_{N, \gamma}$ given by

$$
\begin{aligned}
C_{N, \gamma}= \begin{cases}\left(\gamma+\frac{N}{2}-1\right)^{2} \frac{N+1+\left(\gamma-\frac{N}{2}\right)^{2}}{N-1+\left(\gamma-\frac{N}{2}\right)^{2}} & (N \geq 3, \gamma \leq 1), \\
\left(\gamma+\frac{N}{2}-1\right)^{2}+2+\min _{\kappa \geq 0}\left(\kappa+\frac{4(N-1)(\gamma-1)}{\kappa+N-1+\left(\gamma-\frac{N}{2}\right)^{2}}\right) & (N \geq 4, \gamma>1), \\
\left(\gamma+\frac{1}{2}\right)^{2}+2 & (N=3, \gamma>1),\end{cases} \\
C_{2, \gamma}= \begin{cases}\gamma^{2} \frac{3+(\gamma-1)^{2}}{1+(\gamma-1)^{2}} & \text { if }|\gamma+1| \leq \sqrt{3}, \\
\gamma^{2}+1 & \text { otherwise. }\end{cases}
\end{aligned}
$$

Note that the expression of the best constant $C_{N, \gamma}$ is slightly different from that in [2] when $N \geq 4$, but a careful checking the proof in [2] leads to the above formula in Theorem A. (See also [3, §2.1].)

Later, the first author of this paper has succeeded in removing the axisymmetric

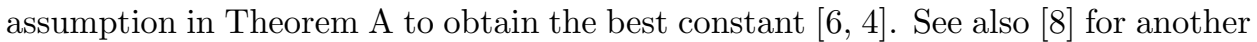
improvement of [2]. We refer to [3, 5] for the Rellich-Leray inequality for divergencefree vector fields.

For curl-free vector fields, we have recently obtained the following result.

Theorem B. ([[]]) Let $N \geq 2$. Let $\gamma \neq 1-N / 2$ be a real number and let $\boldsymbol{u} \in$ $C_{c}^{\infty}\left(\mathbb{R}^{N}\right)^{N}$ be a curl-free vector field. We assume that $\boldsymbol{u}(\mathbf{0})=\mathbf{0}$ if $\gamma<1-N / 2$. Then

$$
H_{N, \gamma} \int_{\mathbb{R}^{N}} \frac{|\boldsymbol{u}|^{2}}{|\boldsymbol{x}|^{2}}|\boldsymbol{x}|^{2 \gamma} d x \leq \int_{\mathbb{R}^{N}}|\nabla \boldsymbol{u}|^{2}|\boldsymbol{x}|^{2 \gamma} d x
$$

with the optimal constant $H_{N, \gamma}$ given by

$$
H_{N, \gamma}= \begin{cases}\left(\gamma+\frac{N}{2}-1\right)^{2} \frac{3(N-1)+\left(\gamma+\frac{N}{2}-2\right)^{2}}{N-1+\left(\gamma+\frac{N}{2}-2\right)^{2}} & \text { if }\left|\gamma+\frac{N}{2}\right| \leq \sqrt{N+1} \\ \left(\gamma+\frac{N}{2}-1\right)^{2}+N-1 & \text { otherwise. }\end{cases}
$$

The method of the proof of Theorem B, which followed from that of CostinMaz'ya [2], consists of the following items: A representation of curl-free vector fields in the spherical polar coordinates, a transformation of vector fields called BrezisVázquez-Maz'ya, the one-dimensional Fourier transform in the radial direction, and the eigenvector expansion for the Laplace-Beltrami operator in $L^{2}\left(\mathbb{S}^{N-1}\right)$.

A main purpose of this paper is to give another and a simpler approach to Theorem B. We avoid the use of Fourier transform, in the hope of being helpful for the possible extension of the result to $L^{p}$-setting or to domains other than the whole space. As a by-product, we obtain the sharp Hardy-Leray inequality for curl-free vector fields with a remainder term, which is the main result of this paper:

Theorem 1. Let $N \geq 2$. Let $H_{N, \gamma}$ be defined in (⿴囗十) and let $\boldsymbol{u} \in C_{c}^{\infty}\left(\mathbb{R}^{N}\right)^{N}$ be a curl-free field such that $\boldsymbol{u}(\mathbf{0})=\mathbf{0}$ if $\gamma \leq 1-\frac{N}{2}$. Then the inequality

$$
\begin{aligned}
\int_{\mathbb{R}^{N}}|\nabla \boldsymbol{u}|^{2}|\boldsymbol{x}|^{2 \gamma} d x \geq & H_{N, \gamma} \int_{\mathbb{R}^{N}}|\boldsymbol{u}|^{2}|\boldsymbol{x}|^{2 \gamma-2} d x \\
& +\int_{\mathbb{R}^{N}}\left((N-1) \mathcal{E}_{N, \gamma}[\boldsymbol{u}]+\left|\boldsymbol{x} \cdot \nabla\left(|\boldsymbol{x}|^{\gamma+\frac{N}{2}-1} \boldsymbol{u}\right)\right|^{2}\right)|\boldsymbol{x}|^{-N} d x
\end{aligned}
$$


holds with the nonnegative function $\mathcal{E}_{N, \gamma}[\boldsymbol{u}]$ given by

$$
\begin{aligned}
\mathcal{E}_{N, \gamma}[\boldsymbol{u}] & (\boldsymbol{x}) \\
= & \begin{cases}\left(\left(\gamma+\frac{N}{2}\right)^{2}-N-1\right) \varphi^{2}+(\boldsymbol{x} \cdot \nabla \varphi)^{2} & \text { for }\left|\gamma+\frac{N}{2}\right| \geq \sqrt{N+1}, \\
\frac{\left(N+1-\left(\gamma+\frac{N}{2}\right)^{2}\right) f^{2}+4(1-\gamma)(\boldsymbol{x} \cdot \nabla \varphi)^{2}}{\left(\gamma+\frac{N}{2}-2\right)^{2}+N-1} & \text { for }\left|\gamma+\frac{N}{2}\right|<\sqrt{N+1 .}\end{cases}
\end{aligned}
$$

Here $f$ and $\varphi$ are scalar fields defined by

$$
\begin{aligned}
& f(\boldsymbol{x})=\omega_{N-1}^{-1}|\boldsymbol{x}|^{\gamma+\frac{N}{2}-1} \int_{\mathbb{S}^{N-1}} \boldsymbol{\sigma} \cdot \boldsymbol{u}(|\boldsymbol{x}| \boldsymbol{\sigma}) \mathrm{d} \sigma, \\
& \varphi(\boldsymbol{x})=|\boldsymbol{x}|^{\gamma+\frac{N}{2}-2}\left(\phi(\boldsymbol{x})-\omega_{N-1}^{-1} \int_{\mathbb{S}^{N-1}} \phi(|\boldsymbol{x}| \boldsymbol{\sigma}) \mathrm{d} \sigma\right),
\end{aligned}
$$

in terms of the scalar potential $\phi$ of $\boldsymbol{u}$ (that is, $\boldsymbol{u}=\nabla \phi$ ), and $\omega_{N-1}$ denotes the surface measure of the unit sphere $\mathbb{S}^{N-1}$ in $\mathbb{R}^{N}$. Moreover, the equality in (四) is realized if and only if the equation

$$
-\triangle_{\sigma} \varphi(r \boldsymbol{\sigma})=(N-1) \varphi(r \boldsymbol{\sigma})
$$

holds for all $r>0$ and $\boldsymbol{\sigma} \in \mathbb{S}^{N-1}$, where $\triangle_{\sigma}$ denotes the Laplace-Beltrami operator on $\mathbb{S}^{N-1}$.

Remark 2. We directly see from (『) that the equation

$$
\int_{\mathbb{R}^{N}}|\nabla \boldsymbol{u}|^{2}|\boldsymbol{x}|^{2 \gamma} d x=H_{N, \gamma} \int_{\mathbb{R}^{N}} \frac{|\boldsymbol{u}|^{2}}{|\boldsymbol{x}|^{2}}|\boldsymbol{x}|^{2 \gamma} d x
$$

does not hold for any $\boldsymbol{u} \in C_{c}^{\infty}\left(\mathbb{R}^{N}\right)^{N} \backslash\{\mathbf{0}\}$ as far as the integral on the right-hand side is finite. Indeed, this equation together with (『) implies that the function

$$
\mathbb{R}_{+} \times \mathbb{S}^{N-1} \ni(r, \boldsymbol{\sigma}) \mapsto r^{\gamma+\frac{N}{2}-1} \boldsymbol{u}(r \boldsymbol{\sigma})
$$

is independent of $r$, which violates the finiteness of the integral unless $\boldsymbol{u} \equiv \mathbf{0}$.

The remaining content of this paper is organized as follows: In $\S$ [ు, we give a quick review of some differential formulae with respect to radial-spherical variables and derive an equivalent condition to the curl-free condition for vector-fields on $\mathbb{R}^{N}$ (the Poincaré lemma); there Proposition $⿴$ gives a characterization of curl-free fields, which serves as a key tool for the proof of our main theorem. In §⿴囗玉 we

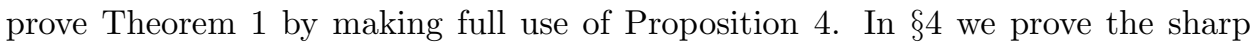
Rellich-Leray inequality for curl-free vector fields with a remainder term, as another application of the method described in $\oint$ 四 $\delta$.

\section{REPRESENTATION OF CURL-FREE FIELDS IN TERMS OF RADIAL-SPHERICAL \\ VARIABLES}

In this section, we recall the Poincaré lemma, which gives a scalar-potential representation of smooth curl-free fields on $\mathbb{R}^{N}$. By deforming this potential via Brezis-Vázquez-Maz'ya transformation, we derive another equivalent condition for test vector fields to be curl-free.

2.1. Radial-spherical variables and the Poincaré lemma. First of all, we introduce the transformation

$$
\mathbb{R}_{+} \times \mathbb{S}^{N-1} \rightarrow \mathbb{R}^{N} \backslash\{\mathbf{0}\}, \quad(r, \boldsymbol{\sigma}) \mapsto \boldsymbol{x}=r \boldsymbol{\sigma}
$$

together with its inverse

$$
\mathbb{R}^{N} \backslash\{\mathbf{0}\} \rightarrow \mathbb{R}_{+} \times \mathbb{S}^{N-1}, \quad \boldsymbol{x} \mapsto(r, \boldsymbol{\sigma})=\left(|\boldsymbol{x}|, \frac{\boldsymbol{x}}{|\boldsymbol{x}|}\right) \in \mathbb{R}_{+} \times \mathbb{S}^{N-1} .
$$


Let $\boldsymbol{u}=\left(u_{1}, u_{2}, \cdots, u_{N}\right): \mathbb{R}^{N} \backslash\{\mathbf{0}\} \rightarrow \mathbb{R}^{N}$ be a vector field. Then its radial scalar component $u_{R}=u_{R}(\boldsymbol{x})$ and spherical vector part $\boldsymbol{u}_{S}=\boldsymbol{u}_{S}(\boldsymbol{x})$ are defined by the formulae

$$
\boldsymbol{u}=\boldsymbol{\sigma} u_{R}+\boldsymbol{u}_{S}, \quad \boldsymbol{\sigma} \cdot \boldsymbol{u}_{S}=0
$$

for all $\boldsymbol{x}=r \boldsymbol{\sigma} \in \mathbb{R}^{N} \backslash\{\mathbf{0}\}$. In a similar way, we denote by $\partial_{r}$ and $\nabla_{\sigma}$ the radial derivative and the spherical gradient, respectively:

$$
\partial_{r} f=\boldsymbol{\sigma} \cdot \nabla f, \quad \nabla_{\sigma} f=r(\nabla f)_{S}
$$

for all $f=f(\boldsymbol{x}) \in C^{\infty}\left(\mathbb{R}^{N} \backslash\{\mathbf{0}\}\right)$, or equivalently

$$
\nabla=\boldsymbol{\sigma} \partial_{r}+\frac{1}{r} \nabla_{\sigma}, \quad \boldsymbol{\sigma} \cdot \nabla_{\sigma}=0 .
$$

The Laplace operator $\triangle=\sum_{k=1}^{N} \partial^{2} / \partial x_{k}^{2}$ is known to be represented in terms of radial-spherical variables by the formula

$$
\triangle=\frac{1}{r^{N-1}} \partial_{r}\left(r^{N-1} \partial_{r}\right)+\frac{1}{r^{2}} \triangle_{\sigma},
$$

where $\triangle_{\sigma}$ denotes the Laplace-Beltrami operator on $\mathbb{S}^{N-1}$. In the following, we use a convention with some ambiguity that for smooth scalar fields and vector fields on $\mathbb{R}^{N} \backslash\{\mathbf{0}\}$, we think of them as functions of $\boldsymbol{\sigma} \in \mathbb{S}^{N-1}$ for $r=|\boldsymbol{x}|$ fixed, when we apply $\nabla_{\sigma}$ or $\triangle_{\sigma}$ to them. As a simple example, the operation of (四) and (四) on the scalar field $r=|\boldsymbol{x}|$ or its powers gives

$$
\nabla r=\sigma \quad \text { and } \quad \Delta r^{s}=\alpha_{s} r^{s-2}, \quad \text { where } \quad \alpha_{s}=s(s+N-2)
$$

for all $s \in \mathbb{R}$.

For later use, we prove the following lemma:

Lemma 3. For any $f \in C^{\infty}\left(\mathbb{S}^{N-1}\right)$,

$$
\left\{\begin{array}{l}
\triangle_{\sigma}(\boldsymbol{\sigma} f)-\boldsymbol{\sigma} \triangle_{\sigma} f=\left(2 \nabla_{\sigma}-(N-1) \boldsymbol{\sigma}\right) f \\
\triangle_{\sigma} \nabla_{\sigma} f-\nabla_{\sigma} \triangle_{\sigma} f=\left((N-3) \nabla_{\sigma}-2 \boldsymbol{\sigma} \triangle_{\sigma}\right) f
\end{array}\right.
$$

holds for all $\boldsymbol{\sigma} \in \mathbb{S}^{N-1}$.

Proof. Take any $f \in C^{\infty}\left(\mathbb{S}^{N-1}\right)$. We identify $f$ with $\tilde{f} \in C^{\infty}\left(\mathbb{R}^{N} \backslash\{\mathbf{0}\}\right)$ by the formula $\tilde{f}(\boldsymbol{x})=f(\boldsymbol{\sigma})$ where $\boldsymbol{\sigma}=\frac{\boldsymbol{x}}{|\boldsymbol{x}|} \in \mathbb{S}^{N-1}$. Note that $\triangle_{\sigma} \boldsymbol{\sigma}=-(N-1) \boldsymbol{\sigma}$ since

$$
0=\triangle \boldsymbol{x}=\left(\partial_{r}^{2}+\frac{N-1}{r} \partial_{r}+\frac{1}{r^{2}} \triangle_{\sigma}\right)(r \boldsymbol{\sigma})=\frac{N-1}{r} \boldsymbol{\sigma}+\frac{1}{r} \triangle_{\sigma} \boldsymbol{\sigma} .
$$

Thus we compute

$$
\begin{aligned}
\triangle_{\sigma}(\boldsymbol{\sigma} f) & =\left(\triangle_{\sigma} \boldsymbol{\sigma}\right) f+\boldsymbol{\sigma}\left(\triangle_{\sigma} f\right)+2\left(\nabla_{\sigma} f \cdot \nabla_{\sigma}\right) \boldsymbol{\sigma} \\
& =-(N-1) \boldsymbol{\sigma} f+\left(\triangle_{\sigma} f\right) \boldsymbol{\sigma}+2 \nabla_{\sigma} f,
\end{aligned}
$$

where we have used $\left(\nabla_{\sigma} f \cdot \nabla_{\sigma}\right) \boldsymbol{\sigma}=\left(\nabla_{\sigma} f \cdot \nabla\right) \boldsymbol{x}=\nabla_{\sigma} f$. This proves the first identity of (6).

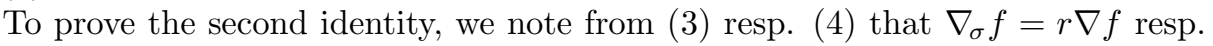
$\triangle_{\sigma} f=r^{2} \triangle f$, since $f=\tilde{f}$ is independent of the radial variable $r$. Also recalling from $(\text { 回 })_{s=1}$ the formulae $\nabla r=\sigma$ and $\triangle r=(N-1) r^{-1}$, we have

$$
\begin{aligned}
\left(\triangle_{\sigma} \nabla_{\sigma}-\nabla_{\sigma} \triangle_{\sigma}\right) f & =r^{2} \triangle(r \nabla f)-r \nabla\left(r^{2} \triangle f\right) \\
& =r^{2}((\triangle r) \nabla f+2(\nabla r \cdot \nabla) \nabla f)-r\left(\nabla r^{2}\right) \triangle f \\
& =(N-1) r \nabla f+2 r^{2} \partial_{r} r^{-1} \nabla_{\sigma} f-2 r^{2} \boldsymbol{\sigma} \triangle f \\
& =(N-3) \nabla_{\sigma} f-2 \boldsymbol{\sigma} \triangle_{\sigma} f,
\end{aligned}
$$

as desired. 
The curl of a vector field $\boldsymbol{u}=\left(u_{1}, \cdots, u_{N}\right) \in C^{\infty}\left(\mathbb{R}^{N}\right)^{N}$ is defined as the differential 2-form

$$
\operatorname{curl} \boldsymbol{u}=d(\boldsymbol{u} \cdot d \boldsymbol{x})=d\left(\sum_{k=1}^{N} u_{k} d x_{k}\right)
$$

where $d$ denotes the exterior differential. This can be expressed in terms of the standard Euclidean coordinates as

$$
d(\boldsymbol{u} \cdot d \boldsymbol{x})=\sum_{k=1}^{N} d u_{k} \wedge d x_{k}=\sum_{j<k} \sum_{j}\left(\frac{\partial u_{k}}{\partial x_{j}}-\frac{\partial u_{j}}{\partial x_{k}}\right) d x_{j} \wedge d x_{k} .
$$

Thus the curl-free condition $d(\boldsymbol{u} \cdot d \boldsymbol{x})=0$ holds if and only if

$$
\frac{\partial u_{k}}{\partial x_{j}}=\frac{\partial u_{j}}{\partial x_{k}} \quad \text { for all } \quad j, k \in\{1, \cdots, N\} .
$$

Here we claim that any curl-free vector fields $\boldsymbol{u}$ can be represented by

$$
\boldsymbol{u}(\boldsymbol{x})=\nabla \phi(\boldsymbol{x}), \quad \phi(\boldsymbol{x})=\int_{0}^{|\boldsymbol{x}|} \frac{\boldsymbol{x}}{|\boldsymbol{x}|} \cdot \boldsymbol{u}\left(\rho \frac{\boldsymbol{x}}{|\boldsymbol{x}|}\right) d \rho \quad \text { for all } \boldsymbol{x} \in \mathbb{R}^{N},
$$

which we say that $\boldsymbol{u}$ has the scalar potential $\phi \in C^{\infty}\left(\mathbb{R}^{N}\right)$. Conversely, the existence of such a potential implies $d(\boldsymbol{u} \cdot d \boldsymbol{x})=d(\nabla \phi \cdot d \boldsymbol{x})=d d \phi=0$, that is, $\boldsymbol{u}$ is curl-free.

The proof of the claim ( $(\mathbb{8})$ is standard: For every $i \in\{1, \cdots, N\}$, we have

$$
\begin{aligned}
u_{i}(\boldsymbol{x}) & =\int_{0}^{1} \frac{d}{d t}\left\{t u_{i}(\boldsymbol{x})\right\} d t=\int_{0}^{1}\left\{u_{i}(t \boldsymbol{x})+t \sum_{j=1}^{N} \frac{\partial u_{i}(t \boldsymbol{x})}{\partial x_{j}} x_{j}\right\} d t \\
& =\int_{0}^{1}\left\{u_{i}(t \boldsymbol{x})+t \sum_{j=1}^{N} \frac{\partial u_{j}(t \boldsymbol{x})}{\partial x_{i}} x_{j}\right\} d t \\
& =\int_{0}^{1} \frac{\partial}{\partial x_{i}}\left(\sum_{j=1}^{N} u_{j}(t \boldsymbol{x}) x_{j}\right) d t=\frac{\partial}{\partial x_{i}} \int_{0}^{1} \boldsymbol{u}(t \boldsymbol{x}) \cdot \boldsymbol{x} d t \quad \forall \boldsymbol{x} \in \mathbb{R}^{N},
\end{aligned}
$$

here we have used (प) in the third equality. Thus we see that $\phi(\boldsymbol{x})=\int_{0}^{1} \boldsymbol{u}(t \boldsymbol{x}) \cdot \boldsymbol{x} d t$ is a scalar potential of $\boldsymbol{u}$. An easy change of variables leads to (घ).

2.2. Radial-spherical decomposition of curl-free fields. In the following, $\lambda \in$ $\mathbb{R}$ denotes a fixed real number. Let $\boldsymbol{u}$ be a curl-free field on $\mathbb{R}^{N}$, and let $\phi$ be its scalar potential ( $\mathbf{\nabla})$. We define a new vector field $\boldsymbol{v}$ and two scalar fields $f, \varphi$ on $\mathbb{R}^{N} \backslash\{\mathbf{0}\}$ by the formulae

$$
\left\{\begin{array}{l}
\boldsymbol{v}(\boldsymbol{x})=|\boldsymbol{x}|^{1-\lambda} \boldsymbol{u}(\boldsymbol{x}), \\
f(\boldsymbol{x})=|\boldsymbol{x}|^{1-\lambda} \omega_{N-1}^{-1} \int_{\mathbb{S}^{N-1}} \boldsymbol{\sigma} \cdot \boldsymbol{u}(|\boldsymbol{x}| \boldsymbol{\sigma}) \mathrm{d} \sigma \\
\varphi(\boldsymbol{x})=|\boldsymbol{x}|^{-\lambda}\left(\phi(\boldsymbol{x})-\omega_{N-1}^{-1} \int_{\mathbb{S}^{N-1}} \phi(|\boldsymbol{x}| \boldsymbol{\sigma}) \mathrm{d} \sigma\right) .
\end{array}\right.
$$

The transformation of the field $\boldsymbol{u} \mapsto \boldsymbol{v}$ by the multiplication of $|\boldsymbol{x}|^{1-\lambda}$ stems from an idea of Brezis-Vázquez [I] and Maz'ya [II]. Now let us denote by

$$
\bar{\phi}(r)=\omega_{N-1}^{-1} \int_{\mathbb{S}^{N-1}} \phi(r \boldsymbol{\sigma}) \mathrm{d} \sigma, \quad r=|\boldsymbol{x}|
$$

the spherical mean of the scalar potential $\phi$ in $(\boldsymbol{\nabla})$, together with its radial derivative

$$
\frac{\partial \bar{\phi}}{\partial r}=\omega_{N-1}^{-1} \int_{\mathbb{S}^{N-1}} \frac{\partial \phi}{\partial r}(r \boldsymbol{\sigma}) \mathrm{d} \sigma=\omega_{N-1}^{-1} \int_{\mathbb{S}^{N-1}}(\boldsymbol{\sigma} \cdot \nabla \phi)(r \boldsymbol{\sigma}) \mathrm{d} \sigma .
$$


Then we see that (प) can be rewritten simply in terms of $\phi$ as

$$
\left\{\begin{array}{l}
\boldsymbol{v}(\boldsymbol{x})=r^{1-\lambda} \nabla \phi(\boldsymbol{x}), \\
f(r)=r^{1-\lambda} \frac{\partial \bar{\phi}}{\partial r}, \\
\varphi(\boldsymbol{x})=r^{-\lambda}(\phi(\boldsymbol{x})-\bar{\phi}(r)),
\end{array}\right.
$$

and that $f$ is a spherical mean part of $r^{1-\lambda} u_{R}$, while $\varphi$ has zero-spherical mean. Furthermore, the scalar representation of $\boldsymbol{u}(\boldsymbol{x})$ in (甘) is transformed into that of $\boldsymbol{v}(\boldsymbol{x})$ by the following computation using (एण):

$$
\begin{aligned}
\boldsymbol{v} & =r^{1-\lambda} \nabla \phi \\
& =r^{1-\lambda}(\nabla(\phi-\bar{\phi})+\nabla \bar{\phi}) \\
& =r^{1-\lambda}\left(\nabla\left(r^{\lambda} \varphi\right)+\frac{\partial \bar{\phi}}{\partial r} \boldsymbol{\sigma}\right) \\
& =r^{1-\lambda} \nabla\left(r^{\lambda} \varphi\right)+f(r) \boldsymbol{\sigma} \\
& =r^{1-\lambda}\left(\lambda r^{\lambda-1} \varphi \boldsymbol{\sigma}+r^{\lambda} \nabla \varphi\right)+f(r) \boldsymbol{\sigma} \\
& =(\lambda \varphi+f) \boldsymbol{\sigma}+r \nabla \varphi \\
& =\left(\lambda \varphi+f+\partial_{t} \varphi\right) \boldsymbol{\sigma}+\nabla_{\sigma} \varphi .
\end{aligned}
$$

Here and hereafter we employ the notation $t=\log r$ which obeys the differential identities

$$
\left\{\begin{array}{l}
\partial_{t}=r \partial_{r}, \quad d t=\frac{d r}{r}, \\
r \nabla=\sigma \partial_{t}+\nabla_{\sigma}, \\
r^{2} \triangle=\partial_{t}^{2}+(N-2) \partial_{t}+\triangle_{\sigma} .
\end{array}\right.
$$

In view of the above computation result, we can say that $f$ and $\varphi$ are radial and spherical scalar potentials of $\boldsymbol{v}$, respectively.

In summary, we obtain the following proposition:

Proposition 4. Let $\lambda \in \mathbb{R}$. Then a vector field $\boldsymbol{u} \in C^{\infty}\left(\mathbb{R}^{N}\right)^{N}$ is curl-free if and only if there exist two scalar fields $f, \varphi \in C^{\infty}\left(\mathbb{R}^{N} \backslash\{\mathbf{0}\}\right)$ satisfying

$$
\left\{\begin{array}{cl}
f \text { is radially symmetric and } \int_{\mathbb{S}^{N-1}} \varphi(r \boldsymbol{\sigma}) \mathrm{d} \sigma=0 & \forall r>0, \\
\boldsymbol{v}=\boldsymbol{\sigma}\left(f+\left(\lambda+\partial_{t}\right) \varphi\right)+\nabla_{\sigma} \varphi & \text { on } \mathbb{R}^{N} \backslash\{\mathbf{0}\}
\end{array}\right.
$$

where $\boldsymbol{v} \in C_{c}^{\infty}\left(\mathbb{R}^{N} \backslash\{\mathbf{0}\}\right)^{N}$ is the vector field given by the same equation $\boldsymbol{v}=r^{1-\lambda} \boldsymbol{u}$ as in (प). Moreover, such $f$ and $\varphi$ are uniquely determined and explicitly given by the equations in (可); in particular, if $\boldsymbol{u}$ has a compact support on $\mathbb{R}^{N} \backslash\{\mathbf{0}\}$, then so do $f$ and $\varphi$.

For later use, we give an expression of the vector field $\triangle_{\sigma} \boldsymbol{v}$ in terms of the scalar potentials:

Lemma 5. Let $\boldsymbol{v}$ be as in (Ш2). Then

$$
\begin{aligned}
\triangle_{\sigma} \boldsymbol{v}= & \boldsymbol{\sigma}\left(\partial_{t}+\lambda-2\right) \triangle_{\sigma} \varphi \\
& +\nabla_{\sigma}\left(2 \partial_{t}+\triangle_{\sigma}+2 \lambda+2 N-4\right) \varphi-(N-1) \boldsymbol{v}
\end{aligned}
$$


Proof. By using Lemma 3 and Proposition 田, we compute

$$
\begin{aligned}
\triangle_{\sigma} \boldsymbol{v}= & \triangle_{\sigma}\left(\boldsymbol{\sigma}\left(f+\left(\partial_{t}+\lambda\right) \varphi\right)\right)+\triangle_{\sigma}\left(\nabla_{\sigma} \varphi\right) \\
= & \left(\boldsymbol{\sigma} \triangle_{\sigma}+2 \nabla_{\sigma}-(N-1) \boldsymbol{\sigma}\right)\left(f+\left(\partial_{t}+\lambda\right) \varphi\right) \\
& +\left(\nabla_{\sigma} \triangle_{\sigma}+(N-3) \nabla_{\sigma}-2 \boldsymbol{\sigma} \triangle_{\sigma}\right) \varphi \\
= & \boldsymbol{\sigma}\left(\left(\partial_{t}+\lambda-2\right) \triangle_{\sigma} \varphi\right)-(N-1) \underbrace{\boldsymbol{\sigma}\left(f+\left(\partial_{t}+\lambda\right) \varphi\right.}_{\boldsymbol{v}-\nabla_{\sigma} \varphi}) \\
& +\nabla_{\sigma}\left(2 \partial_{t}+\triangle_{\sigma}+2 \lambda+N-3\right) \varphi \\
= & \boldsymbol{\sigma}\left(\partial_{t}+\lambda-2\right) \triangle_{\sigma} \varphi \\
& +\nabla_{\sigma}\left(2 \partial_{t}+\triangle_{\sigma}+2 \lambda+2 N-4\right) \varphi-(N-1) \boldsymbol{v} .
\end{aligned}
$$

\section{Proof of Theorem $⿴$}

We assume that the left-hand side of (프) is finite, since otherwise there is nothing to prove. Then the integrability of $|\nabla \boldsymbol{u}|^{2}|\boldsymbol{x}|^{2 \gamma}$ together with the smoothness of $\boldsymbol{u}$ implies the existence of an integer $m>-\frac{N}{2}-\gamma$ such that

$$
\nabla \boldsymbol{u}(\boldsymbol{x})=O\left(|\boldsymbol{x}|^{m}\right) \quad \text { as } \quad|\boldsymbol{x}| \rightarrow 0 .
$$

Moreover, in view of the assumption that $\boldsymbol{u}(\mathbf{0})=\mathbf{0}$ if $\gamma \leq 1-\frac{N}{2}$, we see that $\boldsymbol{u}$ satisfies

$$
|\boldsymbol{x}|^{\gamma+\frac{N}{2}-1} \boldsymbol{u}(\boldsymbol{x})=O\left(|\boldsymbol{x}|^{\varepsilon}\right) \quad \text { as } \quad|\boldsymbol{x}| \rightarrow 0
$$

for $\varepsilon>0$ given by

$$
\varepsilon= \begin{cases}m+\frac{N}{2}+\gamma & \text { for } \gamma \leq 1-\frac{N}{2} \\ \gamma+\frac{N}{2}-1 & \text { for } \gamma>1-\frac{N}{2}\end{cases}
$$

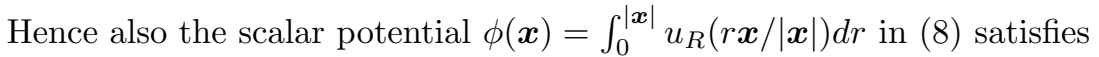

$$
|\boldsymbol{x}|^{\gamma+\frac{N}{2}-1} \phi(\boldsymbol{x})=O\left(|\boldsymbol{x}|^{1+\varepsilon}\right) \quad \text { as }|\boldsymbol{x}| \rightarrow 0 .
$$

Consequently, we have further obtained the integrability conditions

$$
\int_{\mathbb{R}^{N}}|\boldsymbol{u}|^{2}|\boldsymbol{x}|^{2 \gamma-2} d x<\infty \quad \text { and } \quad \int_{\mathbb{R}^{N}} \phi^{2}|\boldsymbol{x}|^{2 \gamma-4} d x<\infty
$$

The proof of the theorem is carried out in the following steps:

3.1. Reduction to the case of compact support distinct from the origin. We can further assume that the curl-free field $\boldsymbol{u}=\nabla \phi$ is compactly supported on $\mathbb{R}^{N} \backslash\{\mathbf{0}\}$ : Indeed, let $\left\{\boldsymbol{u}_{n}\right\} \subset C_{c}^{\infty}\left(\mathbb{R}^{N} \backslash\{\mathbf{0}\}\right)^{N}$ denote the sequence of curl-free fields defined by

$$
\boldsymbol{u}_{n}(\boldsymbol{x})=\nabla\left(\zeta\left(|\boldsymbol{x}|^{\frac{1}{n}}\right) \phi(\boldsymbol{x})\right) \quad \text { for every } n \in \mathbb{N}
$$

where we fix $\zeta \in C_{c}^{\infty}\left(\mathbb{R}_{+}\right)$such that $\zeta(r)=\left\{\begin{array}{ll}0 & \text { for } 0<r<1 / 2 \\ 1 & \text { for } 1 \leq r\end{array}\right.$. Then we see that $\bigcup_{n=1}^{\infty} \operatorname{supp} \boldsymbol{u}_{n}$ is bounded, and that the asymptotic formulae

$$
\begin{aligned}
\boldsymbol{u}_{n}(\boldsymbol{x}) & =\zeta\left(|\boldsymbol{x}|^{\frac{1}{n}}\right) \boldsymbol{u}(\boldsymbol{x})+\boldsymbol{\sigma} n^{-1}|\boldsymbol{x}|^{\frac{1}{n}-1} \zeta^{\prime}\left(|\boldsymbol{x}|^{\frac{1}{n}}\right) \phi(\boldsymbol{x}) \\
& =\boldsymbol{u}(\boldsymbol{x})+o(1) \boldsymbol{u}(\boldsymbol{x})+O(1 / n)|\boldsymbol{x}|^{-1} \phi(\boldsymbol{x}) \\
\nabla \boldsymbol{u}_{n}(\boldsymbol{x}) & =\nabla \boldsymbol{u}(\boldsymbol{x})+o(1) \nabla \boldsymbol{u}(\boldsymbol{x})+O(1 / n) \boldsymbol{\sigma}|\boldsymbol{x}|^{-1} \boldsymbol{u}(\boldsymbol{x})+O(1 / n) \boldsymbol{\sigma} \boldsymbol{\sigma}|\boldsymbol{x}|^{-2} \phi(\boldsymbol{x})
\end{aligned}
$$


hold as $n \rightarrow \infty$. Therefore, taking the $L^{2}$ integration on both sides gives

$$
\begin{aligned}
& \int_{\mathbb{R}^{N}}\left|\boldsymbol{u}_{n}\right|^{2}|\boldsymbol{x}|^{2 \gamma-2} d x=\int_{\mathbb{R}^{N}}|\boldsymbol{u}|^{2}|\boldsymbol{x}|^{2 \gamma-2} d x+o(1), \\
& \int_{\mathbb{R}^{N}}\left|\nabla \boldsymbol{u}_{n}\right|^{2}|\boldsymbol{x}|^{2 \gamma} d x=\int_{\mathbb{R}^{N}}|\nabla \boldsymbol{u}|^{2}|\boldsymbol{x}|^{2 \gamma} d x+o(1),
\end{aligned}
$$

thanks to the integrability conditions (펴). This result shows that the integrals in the inequality $(\boldsymbol{\nabla})$ can be approximated by curl-free fields with compact support on $\mathbb{R}^{N} \backslash\{\mathbf{0}\}$.

3.2. Calculation of the integrals in the Hardy-Leray inequality. In the rest of the present section, we choose

$$
\lambda=2-\frac{N}{2}-\gamma
$$

in view of $\oint$ 2. Then, with respect to the measure

$$
|\boldsymbol{x}|^{2 \gamma} d x=r^{2 \gamma+N-1} d r \mathrm{~d} \sigma=r^{4-2 \lambda} \frac{d r}{r} \mathrm{~d} \sigma=r^{4-2 \lambda} d t \mathrm{~d} \sigma
$$

the $L^{2}$ integration of $\boldsymbol{u}(\boldsymbol{x}) /|\boldsymbol{x}|=r^{\lambda-2} \boldsymbol{v}$ can be expressed in terms of $f$ and $\varphi$ (in Proposition 田) as

$$
\begin{aligned}
\int_{\mathbb{R}^{N}} \frac{|\boldsymbol{u}|^{2}}{|\boldsymbol{x}|^{2}}|\boldsymbol{x}|^{2 \gamma} d x & =\iint_{\mathbb{R} \times \mathbb{S}^{N-1}}|\boldsymbol{v}|^{2} d t \mathrm{~d} \sigma \\
& =\iint_{\mathbb{R} \times \mathbb{S}^{N-1}}\left(\left(f+\partial_{t} \varphi+\lambda \varphi\right)^{2}+\left|\nabla_{\sigma} \varphi\right|^{2}\right) d t \mathrm{~d} \sigma \\
& =\iint_{\mathbb{R}^{\prime} \mathbb{S}^{N-1}}\left(f^{2}+\left(\partial_{t} \varphi\right)^{2}+\lambda^{2} \varphi^{2}+\left|\nabla_{\sigma} \varphi\right|^{2}\right) d t \mathrm{~d} \sigma
\end{aligned}
$$

where the last equality follows from the integration by parts together with the support compactness and $\int_{\mathbb{S}^{N-1}} \varphi \mathrm{d} \sigma=0$. On the other hand, the integration of $|\nabla \boldsymbol{u}|^{2}=\left|\partial_{r} \boldsymbol{u}\right|^{2}+r^{-2}\left|\nabla_{\sigma} \boldsymbol{u}\right|^{2}$ with respect to the measure ([6) yields

$$
\begin{aligned}
\int_{\mathbb{R}^{N}}|\nabla \boldsymbol{u}|^{2}|\boldsymbol{x}|^{2 \gamma} d x & =\int_{\mathbb{R}^{N}}\left(\left|\partial_{r} \boldsymbol{u}\right|^{2}+r^{-2}\left|\nabla_{\sigma} \boldsymbol{u}\right|^{2}\right)|\boldsymbol{x}|^{2 \gamma} d x \\
& =\iint_{\mathbb{R} \times \mathbb{S}^{N-1}}\left(\left|\partial_{r}\left(r^{\lambda-1} \boldsymbol{v}\right)\right|^{2}+r^{-2}\left|\nabla_{\sigma}\left(r^{\lambda-1} \boldsymbol{v}\right)\right|^{2}\right) r^{4-2 \lambda} d t \mathrm{~d} \sigma \\
& =\iint_{\mathbb{R} \times \mathbb{S}^{N-1}}\left(\left|(\lambda-1) \boldsymbol{v}+\partial_{t} \boldsymbol{v}\right|^{2}+\left|\nabla_{\sigma} \boldsymbol{v}\right|^{2}\right) d t \mathrm{~d} \sigma \\
& =\iint_{\mathbb{R} \times \mathbb{S}^{N-1}}\left((\lambda-1)^{2}|\boldsymbol{v}|^{2}+\left|\partial_{t} \boldsymbol{v}\right|^{2}\right) d t \mathrm{~d} \sigma+\iint_{\mathbb{R} \times \mathbb{S}^{N-1}}\left|\nabla_{\sigma} \boldsymbol{v}\right|^{2} d t \mathrm{~d} \sigma .
\end{aligned}
$$


To evaluate the last integral, let us take the $L^{2}$-inner product of $\triangle_{\sigma} \boldsymbol{v}$ in ([ए3) and $\boldsymbol{v}=\boldsymbol{\sigma}\left(f+\left(\partial_{t}+\lambda\right) \varphi\right)+\nabla_{\sigma} \varphi$; then integration by parts gives

$$
\begin{aligned}
\iint_{\mathbb{R} \times \mathbb{S}^{N-1}}\left|\nabla_{\sigma} \boldsymbol{v}\right|^{2} d t \mathrm{~d} \sigma=-\iint_{\mathbb{R} \times \mathbb{S}^{N-1}} \boldsymbol{v} \cdot\left(\triangle_{\sigma} \boldsymbol{v}\right) d t \mathrm{~d} \sigma \\
=-\iint_{\mathbb{R} \times \mathbb{S}^{N-1}}\left(f+\left(\partial_{t}+\lambda\right) \varphi\right)\left(\partial_{t}+\lambda-2\right) \triangle_{\sigma} \varphi d t \mathrm{~d} \sigma \\
\quad+\iint_{\mathbb{R} \times \mathbb{S}^{N-1}}\left(-\nabla_{\sigma} \varphi \cdot \nabla_{\sigma}\left(2 \partial_{t}+\triangle_{\sigma}+2 \lambda+2 N-4\right) \varphi+(N-1)|\boldsymbol{v}|^{2}\right) d t \mathrm{~d} \sigma \\
=\iint_{\mathbb{R} \times \mathbb{S}^{N-1}}\left(\left(\triangle_{\sigma} \varphi\right)^{2}+\left(\lambda^{2}-4 \lambda-2 N+4\right)\left|\nabla_{\sigma} \varphi\right|^{2}\right) d t \mathrm{~d} \sigma \\
\quad+\iint_{\mathbb{R} \times \mathbb{S}^{N-1}}\left(\left|\partial_{t} \nabla_{\sigma} \varphi\right|^{2}+(N-1)|\boldsymbol{v}|^{2}\right) d t \mathrm{~d} \sigma .
\end{aligned}
$$

Here we note that the spectrum of $-\triangle_{\sigma}$ is given by the set

$$
\left\{\alpha_{\nu}=\nu(N+\nu-2) ; \nu \in \mathbb{N} \cup\{0\}\right\},
$$

and hence the estimate

$$
\begin{aligned}
\frac{1}{\int_{\mathbb{S}^{N-1}} \varphi^{2} \mathrm{~d} \sigma} \int_{\mathbb{S}^{N-1}}\left(\left(\triangle_{\sigma} \varphi\right)^{2}\right. & \left.+\left(\lambda^{2}-4 \lambda-2 N+4\right)\left|\nabla_{\sigma} \varphi\right|^{2}\right) \mathrm{d} \sigma \\
& \geq \min _{\nu \in \mathbb{N}}\left\{\alpha_{\nu}^{2}+\left(\lambda^{2}-4 \lambda-2 N+4\right) \alpha_{\nu} ; \nu \in \mathbb{N}\right\} \\
& =\alpha_{1}^{2}+\left(\lambda^{2}-4 \lambda-2 N+4\right) \alpha_{1} \\
& =(N-1)\left((\lambda-2)^{2}-N-1\right)
\end{aligned}
$$

holds for all $\varphi \in C^{\infty}\left(\mathbb{S}^{N-1}\right) \backslash\{0\}$ such that $\int_{\mathbb{S}^{N-1}} \varphi \mathrm{d} \sigma=0$. Also by using $\int_{\mathbb{S}^{N-1}} \partial_{t} \varphi \mathrm{d} \sigma=0$, we have the estimate

$$
\int_{\mathbb{S}^{N-1}}\left|\nabla_{\sigma}\left(\partial_{t} \varphi\right)\right|^{2} \mathrm{~d} \sigma \geq(N-1) \int_{\mathbb{S}^{N-1}}\left|\partial_{t} \varphi\right|^{2} \mathrm{~d} \sigma
$$

as an $L^{2}\left(\mathbb{S}^{N-1}\right)$ version of the Poincaré inequality. Combine the above two estimates with the right-hand side of (एप), and we obtain

$$
\iint_{\mathbb{R} \times \mathbb{S}^{N-1}}\left|\nabla_{\sigma} \boldsymbol{v}\right|^{2} d t \mathrm{~d} \sigma \geq(N-1) \iint_{\mathbb{R} \times \mathbb{S}^{N-1}}\left(|\boldsymbol{v}|^{2}+\left((\lambda-2)^{2}-N-1\right) \varphi^{2}+\left(\partial_{t} \varphi\right)^{2}\right) d t \mathrm{~d} \sigma
$$

to evaluate the last integral in (ㅁ); hence we get

$$
\begin{aligned}
\int_{\mathbb{R}^{N}}|\nabla \boldsymbol{u}|^{2}|\boldsymbol{x}|^{2 \gamma} d x & \\
\geq & \left((\lambda-1)^{2}+N-1\right) \iint_{\mathbb{R} \times \mathbb{S}^{N-1}}|\boldsymbol{v}|^{2} d t \mathrm{~d} \sigma+\iint_{\mathbb{R} \times \mathbb{S}^{N-1}}\left|\partial_{t} \boldsymbol{v}\right|^{2} d t \mathrm{~d} \sigma \\
& +(N-1) \iint_{\mathbb{R} \times \mathbb{S}^{N-1}}\left(\left((\lambda-2)^{2}-N-1\right) \varphi^{2}+\left(\partial_{t} \varphi\right)^{2}\right) d t \mathrm{~d} \sigma .
\end{aligned}
$$

Here the equality holds if and only if $-\triangle_{\sigma} \varphi=\alpha_{1} \varphi$; note that this equation also produces for $\partial_{t} \varphi$ the same equation $-\triangle_{\sigma}\left(\partial_{t} \varphi\right)=\alpha_{1}\left(\partial_{t} \varphi\right)$ since $\partial_{t}$ and $\triangle_{\sigma}$ commutes.

To further proceed, we have the following two cases according to the sign of $(\lambda-2)^{2}-N-1$ : 
3.3. The case $|\lambda-2| \geq \sqrt{N+1}$. Discarding the last two integrals in (更) and recalling the first equation of $(\mathbb{\square})$, we get the Hardy-Leray inequality

$$
\int_{\mathbb{R}^{N}}|\nabla \boldsymbol{u}|^{2}|\boldsymbol{x}|^{2 \gamma} d x \geq H_{N, \gamma} \int_{\mathbb{R}^{N}} \frac{|\boldsymbol{u}|^{2}}{|\boldsymbol{x}|^{2}}|\boldsymbol{x}|^{2 \gamma} d x
$$

for curl-free fields $\boldsymbol{u}$, with the constant

$$
H_{N, \gamma}=(\lambda-1)^{2}+N-1=\left(\gamma+\frac{N}{2}-1\right)^{2}+N-1
$$

To show that this number is the best possible, let us choose the sequence of curl-free fields $\left\{\boldsymbol{u}_{n}=r^{\lambda-1} \boldsymbol{v}_{n}\right\}_{n \in \mathbb{N}} \subset C_{c}^{\infty}\left(\mathbb{R}^{N} \backslash\{\mathbf{0}\}\right)^{N}$ by the formula

$$
\boldsymbol{v}_{n}=\boldsymbol{\sigma} h\left(\frac{t}{n}\right) \quad\left(\text { or equivalently } \boldsymbol{u}_{n}(\boldsymbol{x})=\boldsymbol{x}|\boldsymbol{x}|^{\lambda-2} h\left(\log |\boldsymbol{x}|^{\frac{1}{n}}\right)\right)
$$

for all $(t, \boldsymbol{\sigma}) \in \mathbb{R} \times \mathbb{S}^{N-1}$, where $h \in C_{c}^{\infty}(\mathbb{R})$ such that $h \not \equiv 0$. Then, noticing that the triplet $(\boldsymbol{u}, \boldsymbol{v}, \varphi)=\left(\boldsymbol{u}_{n}, \boldsymbol{v}_{n}, 0\right)$ attains the equality of the inequality (201), we get

$$
\begin{aligned}
\frac{\int_{\mathbb{R}^{N}}\left|\nabla \boldsymbol{u}_{n}\right|^{2}|\boldsymbol{x}|^{2 \gamma} d x}{\int_{\mathbb{R}^{N}}\left|\boldsymbol{u}_{n}\right|^{2}|\boldsymbol{x}|^{2 \gamma-2} d x} & =H_{N, \gamma}+\frac{\iint_{\mathbb{R} \times \mathbb{S}^{N-1}}\left|\partial_{t} \boldsymbol{v}_{n}\right|^{2} d t \mathrm{~d} \sigma}{\iint_{\mathbb{R} \times \mathbb{S}^{N-1}}\left|\boldsymbol{v}_{n}\right|^{2} d t \mathrm{~d} \sigma}=H_{N, \gamma}+\frac{1}{n^{2}} \frac{\int_{\mathbb{R}}\left(h^{\prime}(t)\right)^{2} d t}{\int_{\mathbb{R}}(h(t))^{2} d t} \\
& \longrightarrow H_{N, \gamma} \quad \text { as } n \rightarrow \infty,
\end{aligned}
$$

which proves the best possibility of $H_{N, \gamma}$.

3.4. The case $|\lambda-2|<\sqrt{N+1}$. By using the $L^{2}\left(\mathbb{S}^{N-1}\right)$-Poincaré inequality and equation (ए7), we have

$$
\begin{aligned}
\iint_{\mathbb{R} \times \mathbb{S}^{N-1}} \varphi^{2} d t \mathrm{~d} \sigma & \leq \frac{1}{\lambda^{2}+\alpha_{1}} \iint_{\mathbb{R} \times \mathbb{S}^{N-1}}\left(\lambda^{2} \varphi^{2}+\left|\nabla_{\sigma} \varphi\right|^{2}\right) d t \mathrm{~d} \sigma \\
& =\frac{1}{\lambda^{2}+N-1} \iint_{\mathbb{R} \times \mathbb{S}^{N-1}}\left(|\boldsymbol{v}|^{2}-\left(f^{2}+\left(\partial_{t} \varphi\right)^{2}\right)\right) d t \mathrm{~d} \sigma
\end{aligned}
$$

where the first equality holds if and only if $-\triangle_{\sigma} \varphi=\alpha_{1} \varphi$. Combining this estimate with (201) and noting that $(\lambda-2)^{2}-N-1<0$, we get

$$
\begin{aligned}
\int_{\mathbb{R}^{N}}|\nabla \boldsymbol{u}|^{2}|\boldsymbol{x}|^{2 \gamma} d x & \\
\geq & \iint_{\mathbb{R} \times \mathbb{S}^{N-1}}\left(\left((\lambda-1)^{2}+N-1\right)|\boldsymbol{v}|^{2}+\left|\partial_{t} \boldsymbol{v}\right|^{2}+(N-1)\left(\partial_{t} \varphi\right)^{2}\right) d t \mathrm{~d} \sigma \\
& -\frac{(N-1)\left(N+1-(\lambda-2)^{2}\right)}{\lambda^{2}+N-1} \iint_{\mathbb{R} \times \mathbb{S}^{N-1}}\left(|\boldsymbol{v}|^{2}-\left(f^{2}+\left(\partial_{t} \varphi\right)^{2}\right)\right) d t \mathrm{~d} \sigma \\
= & \frac{(\lambda-1)^{2}\left(\lambda^{2}+3(N-1)\right)}{\lambda^{2}+N-1} \iint_{\mathbb{R} \times \mathbb{S}^{N-1}}|\boldsymbol{v}|^{2} d t \mathrm{~d} \sigma+\int \underset{\mathbb{R} \times \mathbb{S}^{N-1}}{\left.\int \partial_{t} \boldsymbol{v}\right|^{2} d t \mathrm{~d} \sigma} \\
& +(N-1) \iint_{\mathbb{R} \times \mathbb{S}^{N-1}}\left(\frac{N+1-(\lambda-2)^{2}}{\lambda^{2}+N-1} f^{2}+\frac{4\left(\lambda+\frac{N}{2}-1\right)}{\lambda^{2}+N-1}\left(\partial_{t} \varphi\right)^{2}\right) d t \mathrm{~d} \sigma,
\end{aligned}
$$

where the first equality holds if and only if $-\triangle_{\sigma} \varphi=\alpha_{1} \varphi$. In the same way as

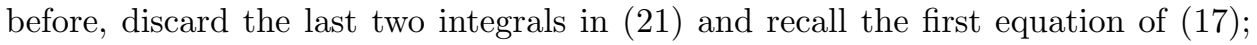
then the Hardy-Leray inequality for curl-free fields

$$
\int_{\mathbb{R}^{N}}|\nabla \boldsymbol{u}|^{2}|\boldsymbol{x}|^{2 \gamma} d x \geq H_{N, \gamma} \int_{\mathbb{R}^{N}} \frac{|\boldsymbol{u}|^{2}}{|\boldsymbol{x}|^{2}}|\boldsymbol{x}|^{2 \gamma} d x
$$


holds with the constant $H_{N, \gamma}$ given by

$$
H_{N, \gamma}=\frac{(\lambda-1)^{2}\left(\lambda^{2}+3(N-1)\right)}{\lambda^{2}+N-1}=\left(\gamma+\frac{N}{2}-1\right)^{2} \frac{\left(\gamma+\frac{N}{2}-2\right)^{2}+3(N-1)}{\left(\gamma+\frac{N}{2}-2\right)^{2}+N-1} .
$$

To show that this $H_{N, \gamma}$ is sharp, let us choose the sequence of curl-free fields $\left\{\boldsymbol{u}_{n}=r^{\lambda-1} \boldsymbol{v}_{n}\right\}_{n \in \mathbb{N}} \subset C_{c}^{\infty}\left(\mathbb{R}^{N} \backslash\{\mathbf{0}\}\right)^{N}$ by the formulae

$$
\left\{\begin{array}{l}
\boldsymbol{v}_{n}=\boldsymbol{\sigma}\left(\partial_{t}+\lambda\right) \varphi_{n}+\nabla_{\sigma} \varphi_{n} \quad\left(\text { or equivalently } \boldsymbol{u}_{n}(\boldsymbol{x})=\nabla\left(|\boldsymbol{x}|^{\lambda} \varphi_{n}(\boldsymbol{x})\right)\right) \\
\varphi_{n}=h\left(\frac{t}{n}\right) Y_{1}(\boldsymbol{\sigma}),
\end{array}\right.
$$

for all $(t, \boldsymbol{\sigma}) \in \mathbb{R} \times \mathbb{S}^{N-1}$, where $h \in C_{c}^{\infty}(\mathbb{R}) \backslash\{0\}$ and where $Y_{1} \in C^{\infty}\left(\mathbb{S}^{N-1}\right)$ denotes the eigenfunction of $-\triangle_{\sigma}$ associated with the eigenvalue $\alpha_{1}=N-1$. Then a straightforward calculation yields

$$
\begin{aligned}
\frac{\int_{\mathbb{R} \times \mathbb{S}^{N-1}}\left(\partial_{t} \varphi_{n}\right)^{2} d t \mathrm{~d} \sigma}{\int_{\mathbb{R} \times \mathbb{S}^{N-1}}\left|\boldsymbol{v}_{n}\right|^{2} d t \mathrm{~d} \sigma} & =\frac{\iint_{\mathbb{R} \times \mathbb{S}^{N-1}}\left(\partial_{t} \varphi_{n}\right)^{2} d t \mathrm{~d} \sigma}{\int_{\mathbb{R} \times \mathbb{S}^{N-1}}\left(\left(\lambda^{2}+\alpha_{1}\right) \varphi_{n}^{2}+\left(\partial_{t} \varphi_{n}\right)^{2}\right) d t \mathrm{~d} \sigma} \\
& =\frac{\int_{\mathbb{R}} n^{-2}\left(h^{\prime}(t)\right)^{2} d t}{\int_{\mathbb{R}}\left(\left(\lambda^{2}+\alpha_{1}\right)(h(t))^{2}+n^{-2}\left(h^{\prime}(t)\right)^{2}\right) d t} \underset{(n \rightarrow \infty)}{\longrightarrow} 0, \\
\frac{\int_{\mathbb{R} \times \mathbb{S}^{N-1}}\left|\partial_{t} \boldsymbol{v}_{n}\right|^{2} d t \mathrm{~d} \sigma}{\int_{\mathbb{R} \times \mathbb{S}^{N-1}}\left|\boldsymbol{v}_{n}\right|^{2} d t \mathrm{~d} \sigma} & =\frac{\int_{\mathbb{R} \times \mathbb{S}^{N-1}}\left(\left(\lambda^{2}+\alpha_{1}\right)\left(\partial_{t} \varphi_{n}\right)^{2}+\left(\partial_{t}^{2} \varphi_{n}\right)^{2}\right) d t \mathrm{~d} \sigma}{\int_{\mathbb{R} \times \mathbb{S}^{N-1}}\left(\left(\lambda^{2}+\alpha_{1}\right) \varphi_{n}^{2}+\left(\partial_{t} \varphi_{n}\right)^{2}\right) d t \mathrm{~d} \sigma} \\
& =\frac{\int_{\mathbb{R}}\left(n^{-2}\left(\lambda^{2}+\alpha_{1}\right)\left(h^{\prime}(t)\right)^{2}+n^{-4}\left(h^{\prime \prime}(t)\right)^{2}\right) d t}{\int_{\mathbb{R}}\left(\left(\lambda^{2}+\alpha_{1}\right)(h(t))^{2}+n^{-2}\left(h^{\prime}(t)\right)^{2}\right) d t} \underset{(n \rightarrow \infty)}{\longrightarrow} 0 .
\end{aligned}
$$

Since the quadruple $(\boldsymbol{u}, \boldsymbol{v}, \varphi, f)=\left(\boldsymbol{u}_{n}, \boldsymbol{v}_{n}, \varphi_{n}, 0\right)$ attains the equality in (四), the above calculation directly gives

$$
\begin{aligned}
& \frac{\int_{\mathbb{R}^{N}}\left|\nabla \boldsymbol{u}_{n}\right|^{2}|\boldsymbol{x}|^{2 \gamma} d x}{\int_{\mathbb{R}^{N}}\left|\boldsymbol{u}_{n}\right|^{2}|\boldsymbol{x}|^{2 \gamma-2} d x} \\
& \quad=H_{N, \gamma}+\frac{\iint_{\mathbb{R}^{2} \mathbb{S}^{N-1}}\left(\left|\partial_{t} \boldsymbol{v}_{n}\right|^{2} d t \mathrm{~d} \sigma+\frac{4(N-1)\left(\lambda+\frac{N}{2}-1\right)}{\lambda^{2}+N-1}\left(\partial_{t} \varphi_{n}\right)^{2}\right) d t \mathrm{~d} \sigma}{\iint_{\mathbb{R} \times \mathbb{S}^{N-1}}\left|\boldsymbol{v}_{n}\right|^{2} d t \mathrm{~d} \sigma} \\
& \quad \longrightarrow H_{N, \gamma} \quad \text { as } n \rightarrow \infty, \quad
\end{aligned}
$$

which proves the sharpness of $H_{N, \gamma}$.

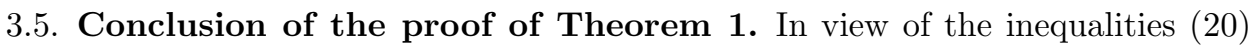

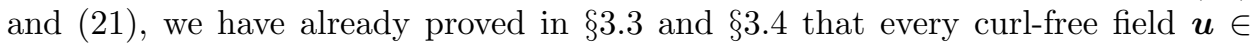
$C_{c}^{\infty}\left(\mathbb{R}^{N} \backslash\{\mathbf{0}\}\right)^{N}$ satisfies the inequality

$$
\begin{aligned}
\int_{\mathbb{R}^{N}}|\nabla \boldsymbol{u}|^{2}|\boldsymbol{x}|^{2 \gamma} d x \geq & H_{N, \gamma} \int_{\mathbb{R}^{N}}|\boldsymbol{u}|^{2}|\boldsymbol{x}|^{2 \gamma-2} d x \\
& +\iint_{\mathbb{R}^{N \mathbb{S}^{N-1}}}\left|\partial_{t} \boldsymbol{v}\right|^{2} d t \mathrm{~d} \sigma+(N-1) \iint_{\mathbb{R}^{\prime} \mathbb{S}^{N-1}} \mathcal{E}_{N, \gamma}[\boldsymbol{u}] d t \mathrm{~d} \sigma
\end{aligned}
$$

with the constant $H_{N, \gamma}$ in Theorem $\mathrm{B}$ and the remainder function $\mathcal{E}_{N, \gamma}[\boldsymbol{u}]$ given by $\mathcal{E}_{N, \gamma}[\boldsymbol{u}](\boldsymbol{x})= \begin{cases}\left((\lambda-2)^{2}-N-1\right) \varphi^{2}+\left(\partial_{t} \varphi\right)^{2} & \text { for }|\lambda-2| \geq \sqrt{N+1}, \\ \frac{N+1-(\lambda-2)^{2}}{\lambda^{2}+N-1} f^{2}+\frac{4\left(\lambda+\frac{N}{2}-1\right)}{\lambda^{2}+N-1}\left(\partial_{t} \varphi\right)^{2} & \text { for }|\lambda-2|<\sqrt{N+1} .\end{cases}$

Moreover, the equality in the above integral inequality holds if and only if $-\triangle_{\sigma} \varphi=$ $\alpha_{1} \varphi$. Finally, restoring the notations

$$
\lambda=2-\frac{N}{2}-\gamma, \quad \partial_{t}=\boldsymbol{x} \cdot \nabla, \quad d t \mathrm{~d} \sigma=|\boldsymbol{x}|^{-N} d x, \quad \boldsymbol{v}=|\boldsymbol{x}|^{\gamma+\frac{N}{2}-1} \boldsymbol{u}
$$


we complete the proof.

\section{A PROOF of the SharP Rellich-LeRAy inequality FOR CURL-FREE} FIELDS

The same approach to prove Theorem $\mathrm{d}$ can also be applied to treat other inequalities involving higher-order derivatives. The following sharp Rellich-Leray inequality for curl-free fields was first proven in [r].

Theorem C. ([[]]) Let $\boldsymbol{u} \in C_{c}^{\infty}\left(\mathbb{R}^{N} \backslash\{\mathbf{0}\}\right)^{N}$ be a curl-free vector field. Then the inequality

$$
R_{N, \gamma} \int_{\mathbb{R}^{N}} \frac{|\boldsymbol{u}|^{2}}{|\boldsymbol{x}|^{4}}|\boldsymbol{x}|^{2 \gamma} d x \leq \int_{\mathbb{R}^{N}}|\triangle \boldsymbol{u}|^{2}|\boldsymbol{x}|^{2 \gamma} d x
$$

holds with the best constant $R_{N, \gamma}$ given by

$$
R_{N, \gamma}=\min \left\{\left(\alpha_{\gamma-\frac{N}{2}}-N+1\right)^{2}, \min _{\nu \in \mathbb{N}} \frac{\left(\gamma+\frac{N}{2}-1\right)^{2}+\alpha_{\nu}}{\left(\gamma+\frac{N}{2}-3\right)^{2}+\alpha_{\nu}}\left(\alpha_{\gamma-\frac{N}{2}-1}-\alpha_{\nu}\right)^{2}\right\}
$$

in terms of the same notation $\alpha_{s}=s(s+N-2)$ as in (回).

In this section, we prove the following improvement of Theorem C.

Theorem 6. Let $R_{N, \gamma}$ be the same as in (ए23). Then the inequality (ए2) can be further improved to be

$$
\int_{\mathbb{R}^{N}}|\triangle \boldsymbol{u}|^{2}|\boldsymbol{x}|^{2 \gamma} d x \geq R_{N, \gamma} \int_{\mathbb{R}^{N}} \frac{|\boldsymbol{u}|^{2}}{|\boldsymbol{x}|^{4}}|\boldsymbol{x}|^{2 \gamma} d x+c_{N, \gamma} \int_{\mathbb{R}^{N}}\left|\boldsymbol{x} \cdot \nabla\left(|\boldsymbol{x}|^{\gamma+\frac{N}{2}-2} \boldsymbol{u}\right)\right|^{2}|\boldsymbol{x}|^{-N} d x
$$

for some positive constant $c_{N, \gamma}>0$.

As a direct consequence of this fact, the equality sign of inequality (E2Z) is never attained by any non-zero curl-free field $\boldsymbol{u}$.

Proof. Let $\boldsymbol{u} \in C_{c}^{\infty}\left(\mathbb{R}^{N} \backslash\{\mathbf{0}\}\right)^{N}$ be a curl-free field. Applying the replacement

$$
\gamma \longmapsto \gamma-1
$$

to equation (며), we choose

$$
\lambda=3-N / 2-\gamma .
$$

By this choice, let us calculate the integrals in inequality (2Z2): Apply the replacement ([24) to the equations in (ㅁ), and we have

$$
\begin{aligned}
\int_{\mathbb{R}^{N}} \frac{|\boldsymbol{u}|^{2}}{|\boldsymbol{x}|^{4}}|\boldsymbol{x}|^{2 \gamma} d x & =\iint_{\mathbb{R} \times \mathbb{S}^{N-1}}|\boldsymbol{v}|^{2} d t \mathrm{~d} \sigma \\
& =\iint_{\mathbb{R} \times \mathbb{S}^{N-1}}\left(f^{2}+\left(\partial_{t} \varphi\right)^{2}+\lambda^{2} \varphi^{2}+\left|\nabla_{\sigma} \varphi\right|^{2}\right) d t \mathrm{~d} \sigma \\
& =\iint_{\mathbb{R} \times \mathbb{S}^{N-1}}\left(f^{2}+\varphi\left(\lambda^{2}-\partial_{t}^{2}-\triangle_{\sigma}\right) \varphi\right) d t \mathrm{~d} \sigma,
\end{aligned}
$$

where the last equality follows from integration by parts together with the support

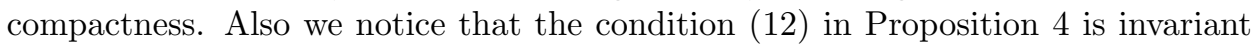
under the following replacement of the triplet:

$$
(\boldsymbol{v}, f, \varphi) \longmapsto\left(\partial_{t}^{k} \boldsymbol{v}, \partial_{t}^{k} f, \partial_{t}^{k} \varphi\right)
$$

for $k=1,2$. Hence we have

$$
\iint_{\mathbb{R} \times \mathbb{S}^{N-1}}\left|\partial_{t}^{k} \boldsymbol{v}\right|^{2} d t \mathrm{~d} \sigma=\iint_{\mathbb{R} \times \mathbb{S}^{N-1}}\left(\left(\partial_{t}^{k} f\right)^{2}+\varphi\left(\lambda^{2}-\partial_{t}^{2}-\triangle_{\sigma}\right)\left(-\partial_{t}^{2}\right)^{k} \varphi\right) d t \mathrm{~d} \sigma .
$$


On the other hand, with the aid of (प्D), we have

$$
\begin{aligned}
\triangle \boldsymbol{u} & =\triangle\left(r^{\lambda-1} \boldsymbol{v}\right)=\left(\triangle r^{\lambda-1}\right) \boldsymbol{v}+2\left(\left(\nabla r^{\lambda-1}\right) \cdot \nabla\right) \boldsymbol{v}+r^{\lambda-1} \triangle \boldsymbol{v} \\
& =\alpha_{\lambda-1} r^{\lambda-3} \boldsymbol{v}+2(\lambda-1) r^{\lambda-3} \partial_{t} \boldsymbol{v}+r^{\lambda-3}\left(\partial_{t}^{2}+(N-2) \partial_{t}+\triangle_{\sigma}\right) \boldsymbol{v} \\
& =r^{\lambda-3}\left(\alpha_{\lambda-1} \boldsymbol{v}+(2 \lambda+N-4) \partial_{t} \boldsymbol{v}+\partial_{t}^{2} \boldsymbol{v}+\triangle_{\sigma} \boldsymbol{v}\right),
\end{aligned}
$$

where in the second line we have used the same formula $\triangle r^{\lambda-1}=\alpha_{\lambda-1} r^{\lambda-3}$ as in (回) $)_{s=\lambda-1}$. Then the $L^{2}$ integration (by parts) of this result yields

$$
\begin{aligned}
\int_{\mathbb{R}^{N}}|\Delta \boldsymbol{u}|^{2}|\boldsymbol{x}|^{2 \gamma} d x= & \iint_{\mathbb{R} \times \mathbb{S}^{N-1}}\left|\alpha_{\lambda-1} \boldsymbol{v}+(2 \lambda+N-4) \partial_{t} \boldsymbol{v}+\partial_{t}^{2} \boldsymbol{v}+\triangle_{\sigma} \boldsymbol{v}\right|^{2} d t \mathrm{~d} \sigma \\
= & \iint_{\mathbb{R} \times \mathbb{S}^{N-1}}\left|\partial_{t}^{2} \boldsymbol{v}\right|^{2} d t \mathrm{~d} \sigma+\left((N-2)^{2}+2 \alpha_{\lambda-1}\right) \iint_{\mathbb{R} \times \mathbb{S}^{N-1}}\left|\partial_{t} \boldsymbol{v}\right|^{2} d t \mathrm{~d} \sigma \\
& +2 \iint_{\mathbb{R} \times \mathbb{S}^{N-1}}\left|\nabla_{\sigma} \partial_{t} \boldsymbol{v}\right|^{2} d t \mathrm{~d} \sigma+\iint_{\mathbb{R} \times \mathbb{S}^{N-1}}\left|\triangle_{\sigma} \boldsymbol{v}+\alpha_{\lambda-1} \boldsymbol{v}\right|^{2} d t \mathrm{~d} \sigma,
\end{aligned}
$$

where the second equality follows from the identity $(2 \lambda+N-4)^{2}-2 \alpha_{\lambda-1}=$ $(N-2)^{2}+2 \alpha_{\lambda-1}$. To calculate the second last integral in (ㅍ), we apply the replacement ([2.5) to the equation in ([प्) :

$$
\text { (28) } \begin{aligned}
\iint_{\mathbb{R} \times \mathbb{S}^{N-1}}\left|\partial_{t} \nabla_{\sigma} \boldsymbol{v}\right|^{2} d t \mathrm{~d} \sigma \\
=\iint_{\mathbb{R} \times \mathbb{S}^{N-1}}\left(\begin{array}{l}
\left(\triangle_{\sigma} \partial_{t} \varphi\right)^{2}+\left|\nabla_{\sigma} \partial_{t}^{2} \varphi\right|^{2}+\left((\lambda-2)^{2}-2 N\right)\left|\nabla_{\sigma} \partial_{t} \varphi\right|^{2} \\
+(N-1)\left|\partial_{t} \boldsymbol{v}\right|^{2}
\end{array}\right) d t \mathrm{~d} \sigma \\
=\iint_{\mathbb{R} \times \mathbb{S}^{N-1}}\left(\begin{array}{l}
\varphi\left(-\triangle_{\sigma}^{2} \partial_{t}^{2}-\triangle_{\sigma} \partial_{t}^{4}+\left((\lambda-2)^{2}-2 N\right) \triangle_{\sigma} \partial_{t}^{2}\right) \varphi \\
+(N-1)\left(\left(\partial_{t} f\right)^{2}+\varphi\left(\lambda^{2}-\partial_{t}^{2}-\triangle_{\sigma}\right)\left(-\partial_{t}^{2} \varphi\right)\right)
\end{array}\right) d t \mathrm{~d} \sigma .
\end{aligned}
$$

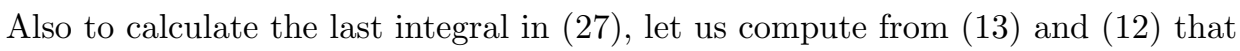

$$
\begin{aligned}
\triangle_{\sigma} \boldsymbol{v}+ & \alpha_{\lambda-1} \boldsymbol{v} \\
= & \boldsymbol{\sigma}\left(\partial_{t}+\lambda-2\right) \triangle_{\sigma} \varphi+\nabla_{\sigma}\left(2 \partial_{t}+\triangle_{\sigma}+2(\lambda+N-2)\right) \varphi \\
& +\left(\alpha_{\lambda-1}-(N-1)\right)\left(\boldsymbol{\sigma}\left(f+\left(\partial_{t}+\lambda\right) \varphi\right)+\nabla_{\sigma} \varphi\right) \\
= & \boldsymbol{\sigma}\left(\left(\triangle_{\sigma}+\alpha_{\lambda-1}-N+1\right) \partial_{t} \varphi+(\lambda-2) \triangle_{\sigma} \varphi+\left(\alpha_{\lambda-1}-N+1\right)(f+\lambda \varphi)\right) \\
& +\nabla_{\sigma}\left(2 \partial_{t}+\triangle_{\sigma}+\alpha_{\lambda}\right) \varphi
\end{aligned}
$$


here we have used $\alpha_{\lambda-1}-(N-1)+2(\lambda+N-2)=\alpha_{\lambda}$ in the second equality. Hence the $L^{2}$ integration by parts of this result yields

$$
\begin{aligned}
\iint_{\mathbb{R} \times \mathbb{S}^{N-1}}\left|\triangle_{\sigma} \boldsymbol{v}+\alpha_{\lambda-1} \boldsymbol{v}\right|^{2} d t \mathrm{~d} \sigma \\
=\iint_{\mathbb{R} \times \mathbb{S}^{N-1}}\left|\begin{array}{l}
\left(\triangle_{\sigma}+\alpha_{\lambda-1}-N+1\right) \partial_{t} \varphi \\
+(\lambda-2) \triangle_{\sigma} \varphi+\left(\alpha_{\lambda-1}-N+1\right)(f+\lambda \varphi)
\end{array}\right| \begin{array}{l}
2 \\
+
\end{array} \\
+\iint_{\mathbb{R} \times \mathbb{S}^{N-1}}\left|\nabla_{\sigma}\left(2 \partial_{t}+\triangle_{\sigma}+\alpha_{\lambda}\right) \varphi\right|^{2} d t \mathrm{~d} \sigma \\
=\iint_{\mathbb{R} \times \mathbb{S}^{N-1}}\left(\begin{array}{l}
+\left|(\lambda-2) \triangle_{\sigma} \varphi+\left(\alpha_{\lambda-1}-N+1\right) \lambda \varphi\right|^{2} \\
+\left|\nabla_{\sigma} \triangle_{\sigma} \varphi+\alpha_{\lambda} \nabla_{\sigma} \varphi\right|^{2}+\left(\alpha_{\lambda-1}-N+1\right)^{2} f^{2}
\end{array}\right) d t \mathrm{~d} \sigma \\
=\iint_{\mathbb{R} \times \mathbb{S}^{N-1}}\left(\begin{array}{l}
\left.\left(\triangle_{\sigma}+\alpha_{\lambda-1}-N+1\right)^{2}-4 \triangle_{\sigma}\right)\left(-\partial_{t}^{2} \varphi\right) \\
+\varphi\left((\lambda-2)^{2}-\triangle_{\sigma}\right)\left(\triangle_{\sigma}+\alpha_{\lambda}\right)^{2} \varphi \\
+\left(\alpha_{\lambda-1}-N+1\right)^{2} f^{2}
\end{array}\right) d t \mathrm{~d} \sigma .
\end{aligned}
$$

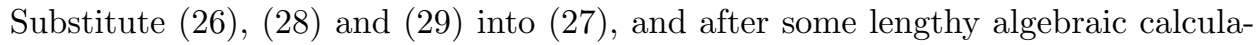
tions, we obtain

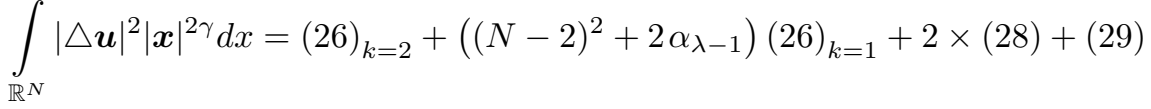

$$
\begin{aligned}
& =\iint_{\mathbb{R} \times \mathbb{S}^{N-1}}\left(\varphi Q_{1}\left(-\partial_{t}^{2},-\triangle_{\sigma}\right) \varphi+f Q_{0}\left(-\partial_{t}^{2}\right) f\right) d t \mathrm{~d} \sigma,
\end{aligned}
$$

where $Q_{1}(\cdot, \cdot)$ and $Q_{0}(\cdot)$ are the polynomials given by

$$
\begin{aligned}
Q_{1}(\tau, \alpha)= & \left(\lambda^{2}+\tau+\alpha\right) \tau^{2}+\left((N-2)^{2}+2 \alpha_{\lambda-1}\right)\left(\lambda^{2}+\tau+\alpha\right) \tau \\
& +2\left(\alpha^{2} \tau+\alpha \tau^{2}+\left((\lambda-2)^{2}-2 N\right) \alpha \tau+(N-1)\left(\lambda^{2}+\tau+\alpha\right) \tau\right) \\
& +\left(\left(-\alpha+\alpha_{\lambda-1}-N+1\right)^{2}+4 \alpha\right) \tau+\left((\lambda-2)^{2}+\alpha\right)\left(-\alpha+\alpha_{\lambda}\right)^{2} \\
= & \left(\tau+\alpha+(\lambda-2)^{2}\right)\left(\begin{array}{l}
\left(\tau+\alpha+\lambda^{2}\right)\left(\tau+\alpha+(\lambda+N-2)^{2}\right) \\
-(2 \lambda+N-2)^{2} \alpha
\end{array}\right) \\
Q_{0}(\tau)= & \tau^{2}+\left((N-2)^{2}+2 \alpha_{\lambda-1}\right) \tau+2(N-1) \tau+\left(\alpha_{\lambda-1}-N+1\right)^{2} \\
= & \left(\tau+(\lambda-2)^{2}\right)\left(\tau+(\lambda+N-2)^{2}\right) .
\end{aligned}
$$

Therefore, we get

$$
\frac{\int_{\mathbb{R}^{N}}|\triangle \boldsymbol{u}|^{2}|\boldsymbol{x}|^{2 \gamma} d x}{\int_{\mathbb{R}^{N}}|\boldsymbol{u}|^{2}|\boldsymbol{x}|^{2 \gamma-4} d x}=\frac{\iint_{\mathbb{R} \times \mathbb{S}^{N-1}}\left(\varphi Q_{1}\left(-\partial_{t}^{2},-\triangle_{\sigma}\right) \varphi+f Q_{0}\left(-\partial_{t}^{2}\right) f\right) d t \mathrm{~d} \sigma}{\iint_{\mathbb{R} \times \mathbb{S}^{N-1}}\left(\varphi\left(\lambda^{2}-\partial_{t}^{2}-\triangle_{\sigma}\right) \varphi+f^{2}\right) d t \mathrm{~d} \sigma}
$$

as far as $\boldsymbol{u} \neq \mathbf{0}$.

From now on, we evaluate the right-hand side of (3:3). We apply to $\varphi$ and $f$ the 1-D Fourier transformation with respect to $t$ : we set

$$
\widehat{\varphi}(\tau, \boldsymbol{\sigma})=\frac{1}{\sqrt{2 \pi}} \int_{\mathbb{R}} e^{i \tau t} \varphi\left(e^{t} \boldsymbol{\sigma}\right) d t, \quad \widehat{f}(\tau)=\frac{1}{\sqrt{2 \pi}} \int_{\mathbb{R}} e^{i \tau t} f\left(e^{t} \boldsymbol{\sigma}\right) d t
$$


for $(\tau, \boldsymbol{\sigma}) \in \mathbb{R} \times \mathbb{S}^{N-1}$. Furthermore, we apply to $\widehat{\varphi}$ the spherical harmonics expansion:

$$
\widehat{\varphi}(\tau, \boldsymbol{\sigma})=\sum_{\nu \in \mathbb{N}} \widehat{\varphi_{\nu}}(\tau) Y_{\nu}(\boldsymbol{\sigma}), \quad\left\{\begin{array}{l}
-\triangle_{\sigma} Y_{\nu}=\alpha_{\nu} Y_{\nu}, \\
\alpha_{\nu}=\nu(\nu+N-2) \quad \forall \nu \in \mathbb{N},
\end{array}\right.
$$

with the normalization $\int_{\mathbb{S}^{N-1}}\left|Y_{\nu}(\boldsymbol{\sigma})\right|^{2} \mathrm{~d} \sigma=1$. Substituting these formulae into ([:3) and noticing the $L^{2}(\mathbb{R})$ isometry of the Fourier transformation, we have

$$
\begin{aligned}
\frac{\int_{\mathbb{R}^{N}}|\Delta \boldsymbol{u}|^{2}|\boldsymbol{x}|^{2 \gamma} d x}{\int_{\mathbb{R}^{N}}|\boldsymbol{u}|^{2}|\boldsymbol{x}|^{2 \gamma-4} d x} & =\frac{\sum_{\nu \in \mathbb{N}} \int_{\mathbb{R}} Q_{1}\left(\tau^{2}, \alpha_{\nu}\right)\left|\widehat{\varphi_{\nu}}(\tau)\right|^{2} d \tau+\omega_{N-1} \int_{\mathbb{R}} Q_{0}\left(\tau^{2}\right)|\widehat{f}(\tau)|^{2} d \tau}{\sum_{\nu \in \mathbb{N}} \int_{\mathbb{R}}\left(\lambda^{2}+\tau^{2}+\alpha_{\nu}\right)\left|\widehat{\varphi_{\nu}}(\tau)\right|^{2} d \tau+\omega_{N-1} \int_{\mathbb{R}}|\widehat{f}(\tau)|^{2} d \tau} \\
& \geq \min \left\{\inf _{\nu \in \mathbb{N}} \inf _{\tau \in \mathbb{R}} \frac{Q_{1}\left(\tau^{2}, \alpha_{\nu}\right)}{\lambda^{2}+\tau^{2}+\alpha_{\nu}}, \inf _{\tau \in \mathbb{R}} Q_{0}\left(\tau^{2}\right)\right\} \\
& =\min \left\{\min _{\nu \in \mathbb{N}} \frac{Q_{1}\left(0, \alpha_{\nu}\right)}{\lambda^{2}+\alpha_{\nu}}, Q_{0}(0)\right\} .
\end{aligned}
$$

Here the last equality follows from that in view of (Bत) and (B2) the functions

$\frac{Q_{1}\left(\tau, \alpha_{\nu}\right)}{\lambda^{2}+\tau+\alpha_{\nu}}=\left(\tau+\alpha_{\nu}+(\lambda-2)^{2}\right)\left(\tau+\alpha_{\nu}\left(1-\frac{(2 \lambda+N-2)^{2}}{\lambda^{2}+\tau+\alpha_{\nu}}\right)+(\lambda+N-2)^{2}\right)$ and $Q_{0}(\tau)$ are monotonically increasing in $\tau \in[0, \infty)$ for each $\nu \in \mathbb{N}$. Therefore, we have proved the Rellich-Leray inequality for curl-free fields ( $(\mathbb{2 Z})$ :

holds with the constant $R_{N, \gamma}$ given by

$$
\int_{\mathbb{R}^{N}}|\triangle \boldsymbol{u}|^{2}|\boldsymbol{x}|^{2 \gamma} d x \geq R_{N, \gamma} \int_{\mathbb{R}^{N}}|\boldsymbol{u}|^{2}|\boldsymbol{x}|^{2 \gamma-4} d x
$$

$$
\begin{aligned}
R_{N, \gamma} & =\min \left\{\min _{\nu \in \mathbb{N}} \frac{Q_{1}\left(0, \alpha_{\nu}\right)}{\lambda^{2}+\alpha_{\nu}}, Q_{0}(0)\right\} \\
& =\min \left\{\min _{\nu \in \mathbb{N}} \frac{(\lambda-2)^{2}+\alpha_{\nu}}{\lambda^{2}+\alpha_{\nu}}\left(\alpha_{\lambda}-\alpha_{\nu}\right)^{2},\left(\alpha_{\lambda-1}-N+1\right)^{2}\right\} \\
& =\min \left\{\min _{\nu \in \mathbb{N}} \frac{\left(\gamma+\frac{N}{2}-1\right)^{2}+\alpha_{\nu}}{\left(\gamma+\frac{N}{2}-3\right)^{2}+\alpha_{\nu}}\left(\alpha_{\gamma-\frac{N}{2}-1}-\alpha_{\nu}\right)^{2},\left(\alpha_{\gamma-\frac{N}{2}}-N+1\right)^{2}\right\} .
\end{aligned}
$$

Now, we prove the sharpness of $R_{N, \gamma}$. For this purpose, we choose $\nu_{0} \in \mathbb{N} \cup\{0\}$ to be such that

$$
\left\{\begin{array}{l}
\nu_{0}=0, \quad \text { if } \min \left\{\min _{\nu \in \mathbb{N}} \frac{Q_{1}\left(0, \alpha_{\nu}\right)}{\lambda^{2}+\alpha_{\nu}}, Q_{0}(0)\right\}=Q_{0}(0), \\
\min _{\nu \in \mathbb{N}} \frac{Q_{1}\left(0, \alpha_{\nu}\right)}{\lambda^{2}+\alpha_{\nu}}=\frac{Q_{1}\left(0, \alpha_{\nu_{0}}\right)}{\lambda^{2}+\alpha_{\nu_{0}}}, \quad \text { otherwise }
\end{array}\right.
$$

and define the sequence of vector fields $\left\{\boldsymbol{u}_{n}=r^{\lambda-1} \boldsymbol{v}_{n}\right\}_{n \in \mathbb{N}} \subset C_{c}^{\infty}\left(\mathbb{R}^{N} \backslash\{\mathbf{0}\}\right)^{N}$ by the formulae

$$
\boldsymbol{v}_{n}= \begin{cases}\boldsymbol{\sigma} f_{n} & \text { if } \nu_{0}=0 \\ \boldsymbol{\sigma}\left(\partial_{t}+\lambda\right) \varphi_{n}+\nabla_{\sigma} \varphi_{n} & \text { otherwise }\end{cases}
$$

Here

$$
\begin{cases}f_{n}(\boldsymbol{x})=h\left(\frac{\log |\boldsymbol{x}|}{n}\right) & \text { if } \nu_{0}=0 \\ \varphi_{n}(\boldsymbol{x})=h\left(\frac{\log |\boldsymbol{x}|}{n}\right) Y_{\nu_{0}}(\boldsymbol{x} /|\boldsymbol{x}|) & \text { otherwise }\end{cases}
$$


with $h \in C_{c}^{\infty}(\mathbb{R}) \backslash\{0\}$, and $Y_{\nu_{0}} \in C^{\infty}\left(\mathbb{S}^{N-1}\right)$ denotes the eigenfunction of $-\triangle_{\sigma}$ associated with the eigenvalue $\alpha_{\nu_{0}}=\nu_{0}\left(\nu_{0}+N-2\right)$. Notice from Proposition $\mathbb{Q}$ that $\boldsymbol{u}_{n}$ is curl-free. Then applying the formula (B.3) to $(\boldsymbol{u}, f, \varphi)=\left(\boldsymbol{u}_{n}, f_{n}, 0\right)$ or $(\boldsymbol{u}, f, \varphi)=\left(\boldsymbol{u}_{n}, 0, \varphi_{n}\right)$ gives

$$
\frac{\int_{\mathbb{R}^{N}}\left|\triangle \boldsymbol{u}_{n}\right|^{2}|\boldsymbol{x}|^{2 \gamma} d x}{\int_{\mathbb{R}^{N}}\left|\boldsymbol{u}_{n}\right|^{2}|\boldsymbol{x}|^{2 \gamma-4} d x}= \begin{cases}\frac{\int_{\mathbb{R}} h\left(\frac{t}{n}\right) Q_{0}\left(-\partial_{t}^{2}\right) h\left(\frac{t}{n}\right) d t}{\int_{\mathbb{R}}\left(h\left(\frac{t}{n}\right)\right)^{2} d t} & \text { if } \nu_{0}=0, \\ \frac{\int_{\mathbb{R}} h\left(\frac{t}{n}\right) Q_{1}\left(-\partial_{t}^{2}, \alpha_{\nu_{0}}\right) h\left(\frac{t}{n}\right) d t}{\int_{\mathbb{R}} h\left(\frac{t}{n}\right)\left(\lambda^{2}-\partial_{t}^{2}+\alpha_{\nu_{0}}\right) h\left(\frac{t}{n}\right) d t} & \text { otherwise. }\end{cases}
$$

Passing to $n \rightarrow \infty$, we get

$$
\begin{aligned}
\frac{\int_{\mathbb{R}^{N}}\left|\triangle \boldsymbol{u}_{n}\right|^{2}|\boldsymbol{x}|^{2 \gamma} d x}{\int_{\mathbb{R}^{N}}\left|\boldsymbol{u}_{n}\right|^{2}|\boldsymbol{x}|^{2 \gamma-4} d x} & =O\left(1 / n^{2}\right)+ \begin{cases}Q_{0}(0) & \text { if } \nu_{0}=0 \\
\frac{Q_{1}\left(0, \alpha_{\nu_{0}}\right)}{\lambda^{2}+\alpha_{\nu_{0}}} & \text { otherwise }\end{cases} \\
& \longrightarrow R_{N, \gamma},
\end{aligned}
$$

which shows the desired sharpness of $R_{N, \gamma}$.

In order to obtain further improvement, we recall that the two integrals in ([2) can be expressed in terms of $\varphi$ and $f$ (in Proposition $甘$ ) as

$$
\begin{aligned}
\int_{\mathbb{R}^{N}}|\triangle \boldsymbol{u}|^{2}|\boldsymbol{x}|^{2 \gamma} d x & =\sum_{\nu \in \mathbb{N}} \int_{\mathbb{R}} Q_{1}\left(\tau^{2}, \alpha_{\nu}\right)\left|\widehat{\varphi_{\nu}}(\tau)\right|^{2} d \tau+\omega_{N-1} \int_{\mathbb{R}} Q_{0}\left(\tau^{2}\right)|\widehat{f}(\tau)|^{2} d \tau, \\
\int_{\mathbb{R}^{N}}|\boldsymbol{u}|^{2}|\boldsymbol{x}|^{2 \gamma-4} d x & =\sum_{\nu \in \mathbb{N}} \int_{\mathbb{R}}\left(\lambda^{2}+\tau^{2}+\alpha_{\nu}\right)\left|\widehat{\varphi_{\nu}}(\tau)\right|^{2} d \tau+\omega_{N-1} \int_{\mathbb{R}}|\widehat{f}(\tau)|^{2} d \tau
\end{aligned}
$$

for $\lambda=3-N / 2-\gamma$, together with the polynomials $Q_{1}$ and $Q_{0}$ given by (Bत) and (अ2). Also recall the expression $R_{N, \gamma}=\min \left\{\min _{\nu \in \mathbb{N}} \frac{Q_{1}\left(0, \alpha_{\nu}\right)}{\lambda^{2}+\alpha_{\nu}}, Q_{0}(0)\right\}$ of the best constant of the inequality $(\overline{22})$ and let $\nu_{1} \in \mathbb{N}$ be such that

$$
\frac{Q_{1}\left(0, \alpha_{\nu_{1}}\right)}{\lambda^{2}+\alpha_{\nu_{1}}}=\min _{\nu \in \mathbb{N}} \frac{Q_{1}\left(0, \alpha_{\nu}\right)}{\lambda^{2}+\alpha_{\nu}} .
$$

Then the difference between the both sides of (ए2) has the following estimate:

$$
\begin{aligned}
\int_{\mathbb{R}^{N}} \mid & \left.\Delta \boldsymbol{u}\right|^{2}|\boldsymbol{x}|^{2 \gamma} d x-\min \left\{\frac{Q_{1}\left(0, \alpha_{\nu_{1}}\right)}{\lambda^{2}+\alpha_{\nu_{1}}}, Q_{0}(0)\right\} \int_{\mathbb{R}^{N}}|\boldsymbol{u}|^{2}|\boldsymbol{x}|^{2 \gamma} d x \\
\geq & \int_{\mathbb{R}^{N}}|\Delta \boldsymbol{u}|^{2}|\boldsymbol{x}|^{2 \gamma} d x \\
& -\frac{Q_{1}\left(0, \alpha_{\nu_{1}}\right)}{\lambda^{2}+\alpha_{\nu_{1}}} \sum_{\nu \in \mathbb{N}} \int_{\mathbb{R}}\left(\tau^{2}+\lambda^{2}+\alpha_{\nu}\right)\left|\widehat{\varphi_{\nu}}(\tau)\right|^{2} d \tau-Q_{0}(0) \omega_{N-1} \int_{\mathbb{R}}|\widehat{f}(\tau)|^{2} d \tau \\
= & \sum_{\nu \in \mathbb{N}} \int_{\mathbb{R}}\left(Q_{1}\left(\tau^{2}, \alpha_{\nu}\right)-\frac{Q_{1}\left(0, \alpha_{\nu_{1}}\right)}{\lambda^{2}+\alpha_{\nu_{1}}}\left(\tau^{2}+\lambda^{2}+\alpha_{\nu}\right)\right)\left|\widehat{\varphi_{\nu}}(\tau)\right|^{2} \\
& +\omega_{N-1} \int_{\mathbb{R}}\left(Q_{0}\left(\tau^{2}\right)-Q_{0}(0)\right)|\widehat{f}(\tau)|^{2} d \tau \\
\geq & c_{1, N, \gamma} \sum_{\nu \in \mathbb{N}} \int_{\mathbb{R}}\left(\lambda^{2}+\tau^{2}+\alpha_{\nu}\right) \tau^{2}\left|\widehat{\varphi_{\nu}}(\tau)\right|^{2} d \tau+c_{0, N, \gamma} \omega_{N-1} \int_{\mathbb{R}} \tau^{2}|\widehat{f}(\tau)|^{2} d \tau \\
\geq & \min \left\{c_{1, N, \gamma}, c_{0, N, \gamma}\right\} \int_{\mathbb{R}}\left(\sum_{\nu \in \mathbb{N}}\left(\lambda^{2}+\tau^{2}+\alpha_{\nu}\right) \tau^{2}\left|\widehat{\varphi_{\nu}}(\tau)\right|^{2}+\omega_{N-1} \tau^{2}|\widehat{f}(\tau)|^{2}\right) d \tau \\
= & \min \left\{c_{1, N, \gamma}, c_{0, N, \gamma}\right\} \int_{\mathbb{R}^{N}}\left|\partial_{t} \boldsymbol{v}\right|^{2} r^{-N} d x,
\end{aligned}
$$


where we have defined the two constants $c_{0, N, \gamma}$ and $c_{1, N, \gamma}$ by

$$
\begin{aligned}
c_{0, N, \gamma} & =\inf _{\tau \in \mathbb{R} \backslash\{0\}} \frac{Q_{0}\left(\tau^{2}\right)-Q_{0}(0)}{\tau^{2}}=Q_{0}^{\prime}(0)=(\lambda-2)^{2}+(\lambda+N-2)^{2} \\
& =N^{2} / 2+2(1-\gamma)^{2}>0, \\
c_{1, N, \gamma} & =\inf _{\nu \in \mathbb{N}} \inf _{\tau \in \mathbb{R} \backslash\{0\}} \frac{1}{\tau^{2}}\left(\frac{Q_{1}\left(\tau^{2}, \alpha_{\nu}\right)}{\tau^{2}+\lambda^{2}+\alpha_{\nu}}-\frac{Q_{1}\left(0, \alpha_{\nu_{1}}\right)}{\lambda^{2}+\alpha_{\nu_{1}}}\right) .
\end{aligned}
$$

Hence it suffices to show $c_{1, N, \gamma}>0$. To this end, notice that

$$
c_{1, N, \gamma} \geq \inf _{\nu \in \mathbb{N}} \inf _{\tau \in \mathbb{R} \backslash\{0\}} \frac{1}{\tau^{2}}\left(\frac{Q_{1}\left(\tau^{2}, \alpha_{\nu}\right)}{\tau^{2}+\lambda^{2}+\alpha_{\nu}}-\frac{Q_{1}\left(0, \alpha_{\nu}\right)}{\lambda^{2}+\alpha_{\nu}}\right)=\inf _{\nu \in \mathbb{N}} \inf _{\tau \geq 0} Q_{2}\left(\tau, \alpha_{\nu}\right)
$$

for the rational polynomial $Q_{2}(\cdot, \cdot)$ defined by the following algebraic calculation:

$$
\begin{aligned}
Q_{2}(\tau, a) & =\frac{1}{\tau}\left(\frac{Q_{1}(\tau, a)}{\tau+\lambda^{2}+a}-\frac{Q_{1}(0, a)}{\lambda^{2}+a}\right) \\
& =\frac{4(1-\lambda)(2 \lambda+N-2)^{2} a}{\left(\lambda^{2}+a\right)\left(\tau+\lambda^{2}+a\right)}+2\left(\lambda+\frac{N}{2}-2\right)^{2}+\frac{N^{2}}{2}+2 a+\tau \\
& =\frac{16(1-\lambda)(2-\gamma)^{2} a}{\left(\lambda^{2}+a\right)\left(\tau+\lambda^{2}+a\right)}+c_{0, N, \gamma}+2 a+\tau
\end{aligned}
$$

In order to further estimate $Q_{2}\left(\tau, \alpha_{\nu}\right)$ for $\tau \geq 0$ and $\nu \in \mathbb{N}$, let us consider the following two cases: for $\lambda \leq 1$, it is clear that $Q_{2}\left(\tau, \alpha_{\nu}\right) \geq c_{0, N, \gamma}+2 \alpha_{\nu}$. For $\lambda>1$, since it is clear that $Q_{2}\left(\tau, \alpha_{\nu}\right)$ is monotone increasing in $\tau$, we have

$$
\begin{aligned}
Q_{2}\left(\tau, \alpha_{\nu}\right) & \geq Q_{2}\left(0, \alpha_{\nu}\right)=-\frac{16(\lambda-1)(2-\gamma)^{2} \alpha_{\nu}}{\left(\lambda^{2}+\alpha_{\nu}\right)^{2}}+c_{0, N, \gamma}+2 \alpha_{\nu} \\
& \geq-\frac{16(\lambda-1)(2-\gamma)^{2} \alpha_{\nu}}{4 \lambda^{2} \alpha_{\nu}}+c_{0, N, \gamma}+2 \alpha_{\nu} \\
& \geq-(2-\gamma)^{2}+c_{0, N, \gamma}+2 \alpha_{\nu} \\
& =\gamma^{2}+N^{2} / 2+2\left(\alpha_{\nu}-1\right) \geq N^{2} / 2+2\left(\alpha_{1}-1\right),
\end{aligned}
$$

where the inequalities in the second and third lines follow from

$$
\left(\lambda^{2}+\alpha_{\nu}\right)^{2} \geq 4 \lambda^{2} \alpha_{\nu} \quad \text { and } \quad-(\lambda-1) / \lambda^{2} \geq-1 / 4 .
$$

Hence it turns out that $\inf _{\nu \in \mathbb{N}} \inf _{\tau \geq 0} Q_{2}\left(\tau, \alpha_{\nu}\right)>0$, and hence that $c_{1, N, \gamma}>0$. Therefore, we have obtained the inequality

$$
\int_{\mathbb{R}^{N}}|\triangle \boldsymbol{u}|^{2}|\boldsymbol{x}|^{2 \gamma} d x-R_{N, \gamma} \int_{\mathbb{R}^{N}}|\boldsymbol{u}|^{2}|\boldsymbol{x}|^{2 \gamma} d x \geq c_{N, \gamma} \int_{\mathbb{R}^{N}}\left|\boldsymbol{x} \cdot \nabla\left(|\boldsymbol{x}|^{\gamma+\frac{N}{2}-1} \boldsymbol{u}\right)\right|^{2}|\boldsymbol{x}|^{-N} d x
$$

for $c_{N, \gamma}=\min \left\{c_{0, N, \gamma}, c_{1, N, \gamma}\right\}>0$. The proof of Theorem $\mathbf{6}$ is now complete, although the constant $c_{N, \gamma}$ is not ensured to be optimal.

\section{Acknowledgments.}

The second author (F.T.) was supported by JSPS Grant-in-Aid for Scientific Research (B), No.19H01800. This work was partly supported by Osaka City University Advanced Mathematical Institute (MEXT Joint Usage/Research Center on Mathematics and Theoretical Physics JPMXP0619217849)). 


\section{REFERENCES}

[1] H. Brezis and J. L. Vázquez, Blow-up solutions of some nonlinear elliptic problems, Revista Matemática de la Universidad Complutense de Madrid 10 (1997), no. 2, 443-469.

[2] O. Costin and V. G. Maz'ya, Sharp Hardy-Leray inequality for axisymmetric divergence-free fields, Calculus of Variations and Partial Differential Equations 32 (2008), no. 4, 523-532.

[3] N. Hamamoto, Sharp Rellich-Leray inequality for axisymmetric divergence-free vector fields, Calculus of Variations and Partial Differential Equations 58 (2019), no. 4, Paper No. 149, 23 pp.

[4] Sharp Hardy-Leray inequality for solenoidal fields, OCAMI Preprint Series (2020).

[5] _ Sharp Rellich-Leray inequality with a radial power weight for solenoidal fields, OCAMI Preprint Series (2020).

[6] _ , Three-dimensional sharp Hardy-Leray inequality for solenoidal fields, Nonlinear Analysis 191 (2020), 111634, 14 pp.

[7] N. Hamamoto and F. Takahashi, Sharp Hardy-Leray and Rellich-Leray inequalities for curlfree vector fields, Mathematische Annalen (2019), to appear.

[8] _ Sharp Hardy-Leray inequality for three-dimensional solenoidal fields with axisymmetric swirl, Communications on Pure \& Applied Analysis 19 (2020), no. 6, 3209-3222.

[9] O. A. Ladyzhenskaya, The mathematical theory of viscous incompressible flow, Mathematics and its Applications, vol. 2, Gordon and Breach, Science Publishers, New York-London-Paris, 1969, Translated from the Russian by Richard A. Silverman and John Chu.

[10] J. Leray, Étude de diverses équations intégrales non linéaires et de quelques problèmes que pose l'hydrodynamique, Journal de Mathématiques Pures et Appliquées 12 (1933), 1-82 (French).

[11] V. G. Maz'ya, Sobolev Spaces with Applications to Elliptic Partial Differential Equations, second, revised and augmented ed., Grundlehren der Mathematischen Wissenschaften [Fundamental Principles of Mathematical Sciences], vol. 342, Springer, Heidelberg, 2011.

Osaka City University Advanced Mathematical Institute,

3-3-138 Sugimoto, Sumiyoshi-ku, Osaka 558-8585, JAPAN

E-mail address: yhjyoe@yahoo.co.jp (N.Hamamoto)

E-mail address: futoshi@sci.osaka-cu.ac.jp (F.Takahashi) 